\title{
Hitting Times of Bessel Processes
}

\author{
T. Byczkowski • J. Małecki • M. Ryznar
}

Received: 1 July 2011 / Accepted: 2 July 2012 / Published online: 15 July 2012

(C) The Author(s) 2012. This article is published with open access at Springerlink.com

\begin{abstract}
Let $T_{1}^{(\mu)}$ be the first hitting time of the point 1 by the Bessel process with index $\mu \in \mathbb{R}$ starting from $x>1$. Using an integral formula for the density $q_{x}^{(\mu)}(t)$ of $T_{1}^{(\mu)}$, obtained in Byczkowski and Ryznar (Stud Math 173(1):19-38, 2006), we prove sharp estimates of the density of $T_{1}^{(\mu)}$ which exhibit the dependence both on time and space variables. Our result provides optimal uniform estimates for the density of the hitting time of the unit ball by the Brownian motion in $\mathbb{R}^{n}$, which improve existing bounds. Another application is to provide sharp estimates for the Poisson kernel for half-spaces for hyperbolic Brownian motion in real hyperbolic spaces.
\end{abstract}

Keywords Bessel process • First hitting time - Geometric Brownian motion • Hyperbolic Brownian motion

Mathematics Subject Classifications (2010) Primary 60J65; Secondary 60J60

\section{Introduction}

Bessel processes play a prominent role both in the theory of Brownian motion (see [18] and [13]) as well as in various theoretical and practical applications. The n-dimensional Bessel process appears quite naturally as the n-dimensional Euclidean norm of Brownian motion; the more intriguing applications are related to the celebrated Ray-Knight theorems describing the behaviour of the local time of Brownian motion in terms of two-dimensional (quadratic) Bessel process (see [21, 23] or [2]). There is also an intimate relation between Bessel processes and the geometric

Research supported by Polish Ministry of Science and Higher Eduction grant N N201 373136.

T. Byczkowski · J. Małecki $(\bowtie) \cdot$ M. Ryznar

Institute of Mathematics and Computer Sciences,

Wrocław University of Technology, Wrocław, Poland

e-mail: jacek.malecki@pwr.wroc.pl 
Brownian motion [15] (the so-called Lamperti's theorem-see Section 2). Bessel processes also appear when representing important jump Lévy processes by means of traces of some multidimensional diffusions (Bessel-Brownian diffusions); see [4, 19]. Another important application consists of the fact that the hyperbolic Brownian motion, i.e. the canonical diffusion in the real hyperbolic space, can be represented as subordination of the standard Brownian motion via an exponential functional of geometric Brownian motion. Thus, from Lamperti's theorem, the Poisson kernel of half-spaces for hyperbolic Brownian motion can be represented via subordination of the standard Brownian motion by the hitting time of a Bessel process (see [5]). We exploit this relationship for obtaining the precise bounds of the Poisson kernel of half-spaces for hyperbolic Brownian motion with drift.

Our main goal is to study estimates of the density of the distribution of the first hitting time $T_{a}^{(\mu)}$ of a level $a>0$ by a Bessel process starting from $x>a$. We do not treat the case $x<a$, since the general theory on the eigenvalue expansions for the first hitting times of diffusion processes (see [14]) may be applied and an explicit simple expression for the density of $T_{a}^{\mu}$ as mixtures of exponential densities involving the zeros of the Bessel function is known. Such a methodology is not applicable in the case $x>a$ due to the lack of compactness property for the corresponding differential operator. Our approach is based on an integral formula for the density of $T_{a}^{(\mu)}$, given in the paper [5] (see also [3]). This formula, although quite complex, proves to be a very effective one. In the proofs we usually deal with processes with nonpositive indices, however our conclusions are valid also for positive ones, since the processes are equivalent on the sigma-algebra generated by the process before hitting 0 . To formulate our results denote by $R^{(\mu)}=\left\{R_{t}^{(\mu)}, t \geqslant 0\right\}$ a Bessel process with index $\mu \in \mathbb{R}$. We denote by $\mathbf{P}_{x}^{(\mu)}$ the probability law of a Bessel process $R^{(\mu)}$ with index $\mu$ on the canonical paths space with starting point $R_{0}^{(\mu)}=x$, where $x>0$. Let us denote the first hitting time of the level $a>0$ by a Bessel process with index $\mu$

$$
T_{a}^{(\mu)}=\inf \left\{t>0 ; R_{t}^{(\mu)}=a\right\} .
$$

Our main result is a sharp estimate of the density of the hitting distribution if $x>a$. By the scaling property of Bessel processes it is enough to consider $x>a=1$. The distribution of $T_{1}^{(\mu)}$ is well known for $\mu= \pm 1 / 2$ when both distributions have the $1 / 2$-stable positive distribution with scale parameter $\lambda=x-1$, however incomplete for $\mu=1 / 2$. Our upper and lower estimates are comparable in both time and space domains. We have the following:

\subsection{Uniform Estimate for a Density Function of $T_{1}^{(\mu)}$}

For every $x>1$ and $t>0$ we have

$$
\frac{\mathbf{P}_{x}^{(\mu)}\left(T_{1}^{(\mu)} \in d t\right)}{d t} \approx(x-1)\left(\frac{1}{1+x^{2 \mu}}\right) \frac{e^{-(x-1)^{2} / 2 t}}{t^{3 / 2}} \frac{x^{2|\mu|-1}}{t^{|\mu|-1 / 2}+x^{|\mu|-1 / 2}}, \quad \mu \neq 0 .
$$

Moreover, we have

$$
\frac{\mathbf{P}_{x}^{(0)}\left(T_{1}^{(0)} \in d t\right)}{d t} \approx(x-1) e^{-(x-1)^{2} / 2 t} \frac{(x+t)^{1 / 2}}{x t^{3 / 2}} \frac{1+\log x}{\left(1+\log \left(1+\frac{t}{x}\right)\right)(1+\log (t+x))} .
$$


Here $f \approx g$ means that there exist strictly positive constants $c_{1}$ and $c_{2}$ depending only on $\mu$ such that $c_{1} \leqslant f / g \leqslant c_{2}$.

Our setup includes the case of the hitting distribution of a unit ball by a Brownian motion starting from the exterior of the ball. To state this result, let $\sigma^{(n)}$ be the first hitting time of a unit ball by $n$-dimensional Brownian motion $W^{(n)}=\left\{W_{t}^{(n)}, t \geqslant 0\right\}$, i.e.

$$
\sigma^{(n)}=\inf \left\{t>0 ;\left|W_{t}^{(n)}\right|=1\right\} .
$$

\subsection{Uniform Estimate for Hitting the Unit Ball by Brownian Motion}

For $W_{0}^{(n)}=x \in \mathbb{R}^{n}$ such that $|x|>1$ we have

$$
\frac{P^{x}\left(\sigma^{(n)} \in d t\right)}{d t} \approx \frac{|x|-1}{|x|} \frac{e^{-(|x|-1)^{2} / 2 t}}{t^{3 / 2}} \frac{1}{t^{(n-3) / 2}+|x|^{(n-3) / 2}}, \quad n>2,
$$

for every $t>0$. Moreover, we have

$$
\frac{P^{x}\left(\sigma^{(2)} \in d t\right)}{d t} \approx \frac{|x|-1}{|x|} e^{-(|x|-1)^{2} / 2 t} \frac{(|x|+t)^{1 / 2}}{t^{3 / 2}} \frac{1+\log |x|}{\left(1+\log \left(1+\frac{t}{|x|}\right)\right)(1+\log (t+|x|))} .
$$

To the best of our knowledge even for the planar Brownian motion our results are new and considerably complement existing results obtained in [10] (see also [6]), where the estimates are only sharp in the region $t \geqslant|x|^{2}$ with sufficiently large starting point $x$. If $t<|x|^{2}$ the bound obtained in [10] has an exponential term of the form $\exp \left\{-c_{i} \frac{|x|^{2}}{t}\right\}$ with different constants $c_{1}, c_{2}$ for the lower and the upper estimate, respectively. We remove this obstacle and provide sharp estimates which are of the same order in the full range of $t$ and $x$. We also provide sharp estimates for the survival probability $\mathbf{P}_{x}^{(\mu)}\left(t<T_{1}^{(\mu)}<\infty\right)$. The asymptotic result if $t \rightarrow \infty$ is due to Hunt [12] in the case of the planar Brownian motion and Port [20] in the context of Brownian motion in higher dimensions. Recently, a result about the asymtotic behaviour of the hitting density for the planar Brownian, when $t \rightarrow \infty$, was established by Uchiyama [22]. His result gives a very accurate expansion of the hitting density provided $|x|^{2}$ is small relative to $t$, that is when the exponential term is negligable. When this is not true the error term of the expansion in [22] may be much bigger than the leading term. Therefore our estimate in the case of the planar Brownian motion is much more accurate, when we assume that $t$ is not too large with respect to $|x|^{2}$, since the impact of the exponential term may be significant and it is reflected in our estimates. Moreover, we give a very exact estimate of the density in the situation when $t$ is small relative to $|x|$ (see Lemma 4 and Remark 1).

The organization of the paper is as follows. After Section 2, in Section 3, we provide uniform estimates of the density function of the first hitting time $T_{1}^{(\mu)}$. This section is basic for further applications, which are collected in the next section. We first provide the estimates of the survival times of a killed Bessel process and, finally, compute the precise bounds of the Poisson kernel of a halfspace for hyperbolic Brownian motion with drift. Appendix contains various estimates of quantities involved in the basic formula for the density function of $T_{1}^{(\mu)}$, which, although quite laborious, but at the same time, are indispensable ingredients of the proof of the main result. 
Throughout the whole paper $f \approx g$ means that there exists a strictly positive constant $c$ depending only on $\mu$ such that $c^{-1} g \leqslant f \leqslant c g$. If the comparability constant will also depend on some other parameters $\gamma_{1}, \gamma_{2}, \ldots$ we will write $f \stackrel{c}{\approx} g$, $c=c\left(\gamma_{1}, \gamma_{2}, \ldots\right)$. Also in a string of inequalities a constant may change from line to line which might not be reflected in notation. Moreover we consistently do not exhibit dependence of $c$ on $\mu$ in inequalities of type $f \leqslant c g$.

\section{Preliminaries}

\subsection{Modified Bessel Functions}

Various formulas appearing throughout the paper are expressed in terms of modified Bessel functions $I_{\vartheta}$ and $K_{\vartheta}$. For convenience we collect here basic information about these functions.

The modified Bessel functions of the first and second kind are independent solutions to the modified Bessel differential equation

$$
z^{2} y^{\prime \prime}+z y^{\prime}-\left(\vartheta^{2}+z^{2}\right) y=0
$$

where $\vartheta \in \mathbb{R}$. The Wronskian of the pair $\left\{K_{\vartheta}(z), I_{\vartheta}(z)\right\}$ is equal to

$$
W\left\{K_{\vartheta}(z), I_{\vartheta}(z)\right\}=I_{\vartheta}(z) K_{\vartheta+1}(z)+I_{\vartheta+1}(z) K_{\vartheta}(z)=\frac{1}{z} .
$$

In the sequel we will use the asymptotic behavior of $I_{\vartheta}$ and $K_{\vartheta}$ at zero as well as at infinity. For every $\vartheta \geqslant 0$ we have (see [1], 9.6.7 and 9.6.12)

$$
I_{\vartheta}(r)=\frac{r^{\vartheta}}{2^{\vartheta} \Gamma(\vartheta+1)}+O\left(r^{\vartheta+2}\right), \quad r \rightarrow 0^{+}, \vartheta>0 .
$$

For $\vartheta>0$, we have ([1], 9.6.9 and 9.6.13)

$$
K_{\vartheta}(r) \cong \frac{2^{\vartheta-1} \Gamma(\vartheta)}{r^{\vartheta}}, \quad r \rightarrow 0^{+},
$$

where $g(r) \cong f(r)$ means that the ratio of $g$ and $f$ tends to 1 . Moreover, in the case $\vartheta=0$, we have (see [1], 9.6.13)

$$
K_{0}(r)=-\log \frac{r}{2} I_{0}(r)+O(1), \quad r \rightarrow 0^{+} .
$$

The behavior of $I_{\vartheta}$ and $K_{\vartheta}$ at infinity is described as follows (see [1], 9.7.1 and 9.7.2)

$$
\begin{aligned}
I_{\vartheta}(r) & =\frac{e^{r}}{\sqrt{2 \pi r}}(1+O(1 / r)), \quad r \rightarrow \infty, \\
K_{\vartheta}(z) & =\sqrt{\frac{\pi}{2 z}} e^{-z}(1+O(1 / z)), \quad|z| \rightarrow \infty,
\end{aligned}
$$

where the last equality is true for every complex $z$ such that $|\arg z|<\frac{3}{2} \pi$. 
2.2 Bessel Process and Exponential Functionals of Brownian Motion

In the following section we introduce notation and basic facts about Bessel processes. We follow the exposition given in [16] and [17], where we refer the reader for more details and deeper insight into the subject (see also [21]).

We denote by $\mathbf{P}_{x}^{(\mu)}$ the probability law of a Bessel process $R^{(\mu)}$ with index $\mu$ on the canonical paths space with starting point $R_{0}^{(\mu)}=x$, where $x>0$. Let $\mathcal{F}_{t}^{(\mu)}=$ $\sigma\left\{R_{s}^{(\mu)}, s \leqslant t\right\}$ be the filtration of the coordinate process $R_{t}^{(\mu)}$. The state space of $R^{(\mu)}$ depends on the value of $\mu$ and the boundary condition at zero. For simplicity, in the case $-1<\mu<0$ (then the point 0 is non-singular), we impose killing condition on 0 . However, the exact boundary condition at 0 is irrelevant from our point of view, because we will only consider the process $R^{(\mu)}$ up to the first hitting time of the strictly positive level.

Let us denote the first hitting time of the level $a>0$ by a Bessel process with index $\mu$ starting from $x>a$

$$
T_{a}^{(\mu)}=\inf \left\{t>0 ; R_{t}^{(\mu)}=a\right\}
$$

Observe that for $\mu \leqslant 0$ we have $T_{a}^{(\mu)}<\infty$ a.s. and $\mathbf{P}_{x}^{(\mu)}\left(T_{a}^{(\mu)}=\infty\right)>0$ whenever $\mu>0$. Using the scaling property of Bessel processes, which is exactly the same as the scaling property of one-dimensional Brownian motion, we get for every $b>0$ and $t>0$

$$
\mathbf{P}_{b x}^{(\mu)}\left(T_{b a}^{(\mu)}<t\right)=\mathbf{P}_{x}^{(\mu)}\left(b^{2} T_{a}^{(\mu)}<t\right), \quad x>a>0 .
$$

Therefore, from now on we do assume that $a=1$ and $x>1$. We denote the density function of $T_{1}^{(\mu)}$ with respect to Lebesgue measure by $q_{x}^{(\mu)}$, i.e.

$$
q_{x}^{(\mu)}(t)=\frac{\mathbf{P}_{x}^{(\mu)}\left(T_{1}^{(\mu)} \in d t\right)}{d t}, \quad t>0, x>1 .
$$

We have the absolute continuity property for the laws of the Bessel processes with different indices

$$
\left.\frac{d \mathbf{P}_{x}^{(\mu)}}{d \mathbf{P}_{x}^{(v)}}\right|_{\mathcal{F}_{t}^{(v)}}=\left(\frac{R_{t}}{x}\right)^{\mu-v} \exp \left(-\frac{\mu^{2}-v^{2}}{2} \int_{0}^{t} \frac{d s}{\left(R_{s}\right)^{2}}\right), \quad \mathbf{P}_{x}^{(v)} \text { - a.s. on }\left\{T_{0}^{(v)}>t\right\} .
$$

Here $T_{0}^{(\mu)}$ denotes the first hitting time of 0 by $R^{(\mu)}$. If $v \geqslant 0$ then the condition $\left\{T_{0}^{(v)}>t\right\}$ can be omitted. In particular, for $v=-\mu$, where $\mu \geqslant 0$ we have

$$
\left.\frac{d \mathbf{P}_{x}^{(\mu)}}{d \mathbf{P}_{x}^{(-\mu)}}\right|_{\mathcal{F}_{t}^{(-\mu)}}=\left(\frac{R_{t}}{x}\right)^{2 \mu}, \quad \mathbf{P}_{x}^{(-\mu)} \text { - a.s. on }\left\{T_{0}^{(-\mu)}>t\right\} .
$$

Consequently, for $\mu \geqslant 0$ and $x>1$ we get that

$$
q_{x}^{(\mu)}(t)=\left(\frac{1}{x}\right)^{2 \mu} q_{x}^{(-\mu)}(t) .
$$


Finally, we recall the relations between Bessel processes and geometric Brownian motion. For more details see [15] and [24]. We denote by $B=\left\{B_{t}, t \geqslant 0\right\}$ the onedimensional Brownian motion starting from 0 and by $B^{(\mu)}=\left\{B_{t}^{(\mu)}=B_{t}+\mu t, t \geqslant 0\right\}$ the Brownian motion with constant drift $\mu \in \mathbb{R}$. The process $X^{(\mu)}=\left\{x \exp \left(B_{t}^{(\mu)}\right)\right.$, $t \geqslant 0\}$ is called a geometric Brownian motion or exponential Brownian motion with drift $\mu \in \mathbb{R}$ starting from $x>0$.

For $x>1$ let $\tau$ be the first exit time of the geometric Brownian motion with drift $\mu$ from the set $(1, \infty)$

$$
\tau=\inf \left\{s>0 ; x \exp \left(B_{s}+\mu s\right)=1\right\} .
$$

We have $\tau<\infty$ a.s. whenever $\mu \leqslant 0$ since then $\inf _{t \geqslant 0} B^{(\mu)}(t)=-\infty$.

For $x>0$ we consider the integral functional

$$
A_{x}^{(\mu)}(t)=\int_{0}^{t}\left(X_{s}^{(\mu)}\right)^{2} d s=x^{2} \int_{0}^{t} \exp \left(2 B_{s}+2 \mu s\right) d s .
$$

The crucial fact which establishes the relation between Bessel processes, the integral functional $A_{x}^{(\mu)}$ and the geometric Brownian motion is the Lamperti relation saying that there exists a Bessel process $R^{(\mu)}$ such that

$$
x \exp \left(B_{t}^{(\mu)}\right)=R_{A_{x}^{(\mu)}(t)}^{(\mu)}, \quad t \geqslant 0 .
$$

Consequently, we get

$$
A_{x}^{(\mu)}(\tau) \stackrel{d}{=} T_{1}^{(\mu)}
$$

\subsection{Representation of Hitting Time Density Function}

We recall the result of [5], where the integral formula for the density of $A_{x}^{(-\mu)}(\tau)$ was given. According to Eq. 9, as immediate consequence, we obtain the formula for $q_{x}^{(-\mu)}(t)$. Note also that in the paper [5] different normalizations of Brownian motion and different definition of geometric Brownian motion were used and consequently we have $q_{x}^{(-\mu)}(t)=q_{\mu}(t / 2) / 2$, where $q_{\mu}(t)$ is the density function considered in [5].

Theorem 1 [5] For $\mu \geqslant 0$ there is a function $w_{\lambda}$ such that

$$
q_{x}^{(-\mu)}(t)=\lambda \frac{e^{-\lambda^{2} / 2 t}}{\sqrt{2 \pi t}}\left(\frac{x^{\mu-1 / 2}}{t}+\int_{0}^{\infty}\left(e^{-\kappa / 2 t}-1\right) w_{\lambda}(v) d v\right),
$$

where $\kappa=\kappa(v)=(\lambda+v)^{2}-\lambda^{2}=v(2 \lambda+v)$, and $\lambda=x-1$.

The function $w_{\lambda}$ appearing in the formulas is described in terms of the modified Bessel functions $K_{\mu}$ and $I_{\mu}$. The function $z \rightarrow K_{\mu}(z)$ extends to an entire function when $\mu-1 / 2$ is an integer and has a holomorphic extension to $\mathbb{C} \backslash(-\infty, 0]$ when $\mu-1 / 2$ is not an integer. Denote the set of zeros of the function $K_{\mu}(z)$ by $Z=\left\{z_{1}, \ldots, z_{k_{\mu}}\right\}$ (cf. [8], p. 62). Recall that $k_{\mu}=\mu-1 / 2$ when $\mu-1 / 2 \in \mathbb{N}$. For $\mu-1 / 2 \notin \mathbb{N}, k_{\mu}$ is the even number closest to $\mu-1 / 2$. The functions $K_{\mu}$ and $K_{\mu-1}$ have no common zeros. 
The function $w_{\lambda}$ is defined as a sum of two functions

$$
w_{\lambda}(v)=w_{1, \lambda}(v)+w_{2, \lambda}(v),
$$

where

$$
w_{1, \lambda}(v)=-\frac{x^{\mu}}{\lambda} \sum_{i=1}^{k_{\mu}} \frac{z_{i} e^{\lambda z_{i}} K_{\mu}\left(x z_{i}\right)}{K_{\mu-1}\left(z_{i}\right)} e^{z_{i} v}
$$

and

$w_{2, \lambda}(v)=-\cos (\pi \mu) \frac{x^{\mu}}{\lambda} \int_{0}^{\infty} \frac{I_{\mu}(x u) K_{\mu}(u)-I_{\mu}(u) K_{\mu}(x u)}{\cos ^{2}(\pi \mu) K_{\mu}^{2}(u)+\left(\pi I_{\mu}(u)+\sin (\pi \mu) K_{\mu}(u)\right)^{2}} e^{-\lambda u} e^{-v u} u d u$.

Moreover the moments of $\kappa$ with respect to $w_{\lambda}(v) d v$ can be computed in the following way

$$
x^{\mu-1 / 2}\left(\mu^{2}-1 / 4\right) / 2 x=\int_{0}^{\infty} w_{\lambda}(v) d v,
$$

and, for $\mu>1 / 2$, we have

$$
2 x^{\mu-1 / 2}=\int_{0}^{\infty} \kappa w_{\lambda}(v) d v
$$

We use also the following representation of $q_{x}^{(-\mu)}(t)$ for $\mu \geqslant 1 / 2$ (see [5], (24))

$$
q_{x}^{(-\mu)}(t)=\lambda \frac{e^{-\lambda^{2} / 2 t}}{\sqrt{2 \pi t}} \int_{0}^{\infty}\left(e^{-\kappa / 2 t}-\sum_{0 \leqslant j \leqslant l}(-1)^{j} \frac{1}{j !}\left(\frac{\kappa}{2 t}\right)^{j}\right) w_{\lambda}(v) d v
$$

where $l=[\mu+1 / 2]$ if $\mu \notin \mathbb{N}$, and $l=\mu-1 / 2$ otherwise.

\section{Uniform Estimates of Hitting Time Density Function}

Throughout of the rest of the paper we denote $\lambda=x-1$. The main result of the paper is the following uniform estimate for a density function of $T_{1}^{(\mu)}$.

Theorem 2 For every $x>1$ and $t>0$ we have

$$
q_{x}^{(\mu)}(t) \approx \lambda\left(\frac{1}{1+x^{2 \mu}}\right) \frac{e^{-\lambda^{2} / 2 t}}{t^{3 / 2}} \frac{x^{2|\mu|-1}}{t^{|\mu|-1 / 2}+x^{|\mu|-1 / 2}}, \quad \mu \neq 0 .
$$

Moreover, we have

$$
q_{x}^{(0)}(t) \approx \begin{cases}\lambda \frac{e^{-\lambda^{2} / 2 t}}{x t} \frac{1+\log x}{\left(1+\log \frac{t}{x}\right)(1+\log t)}, & t>2 x \\ \lambda \frac{e^{-\lambda^{2} / 2 t}}{x^{1 / 2} t^{3 / 2}}, & t \leqslant 2 x\end{cases}
$$

or equivalently

$$
q_{x}^{(0)}(t) \approx \lambda e^{-\lambda^{2} / 2 t} \frac{(x+t)^{1 / 2}}{x t^{3 / 2}} \frac{1+\log x}{\left(1+\log \left(1+\frac{t}{x}\right)\right)(1+\log (t+x))} .
$$


As corollary, putting $\mu=n / 2-1$, we get the corresponding result for $n$ dimensional Brownian motion.

Theorem 3 Let $\sigma^{(n)}$ be the first hitting time of a unit ball by $n$-dimensional Brownian motion $W^{(n)}=\left\{W_{t}^{(n)}, t \geqslant 0\right\}$, i.e.

$$
\sigma^{(n)}=\inf \left\{t>0 ;\left|W_{t}^{(n)}\right|=1\right\} .
$$

Then, for $W_{0}^{(n)}=x \in \mathbb{R}^{n}$ such that $|x|>1$ we have

$$
\frac{P^{x}\left(\sigma^{(n)} \in d t\right)}{d t} \approx \frac{|x|-1}{|x|} \frac{e^{-(|x|-1)^{2} / 2 t}}{t^{3 / 2}} \frac{1}{t^{(n-3) / 2}+|x|^{(n-3) / 2}}, \quad n>2,
$$

for every $t>0$. Moreover, we have

$$
\frac{P^{x}\left(\sigma^{(2)} \in d t\right)}{d t} \approx \frac{|x|-1}{|x|} e^{-(|x|-1)^{2} / 2 t} \frac{(|x|+t)^{1 / 2}}{t^{3 / 2}} \frac{1+\log |x|}{\left(1+\log \left(1+\frac{t}{|x|}\right)\right)(1+\log (t+|x|))}
$$

The proof of the main theorem follows from Lemmas 6-9 given below. We use the crucial estimates of the function $w_{\lambda}(v)$ and its components $w_{1, \lambda}(v)$ and $w_{2, \lambda}(v)$ given in Appendix (see Lemmas 14, 16 and 17).

The following lemma provides satisfactory estimates of $q_{x}^{(-\mu)}(t)$ in the case when $t$ is small relative to $x$.

Lemma 4 We have the following expansion

$$
q_{x}^{(-\mu)}(t)=\lambda \frac{e^{-\lambda^{2} / 4 t}}{(2 \pi)^{1 / 2} t^{3 / 2}} x^{\mu-1 / 2}\left(1+\frac{1-4 \mu^{2}}{8} \frac{t}{x}+E(t, x)\right),
$$

where the error term satisfies the following estimate

$$
|E(t, x)| \leqslant C \frac{t}{x}\left(\sqrt{t} \wedge \frac{t}{\lambda}\right) .
$$

Moreover, for $0 \leqslant \mu<1 / 2$ we have

$$
\lambda \frac{e^{-\lambda^{2} / 2 t}}{(2 \pi)^{1 / 2} t^{3 / 2}} x^{\mu-1 / 2} \leqslant q_{x}^{(-\mu)}(t) \leqslant \lambda \frac{e^{-\lambda^{2} / 4 t}}{(2 \pi)^{1 / 2} t^{3 / 2}} x^{\mu-1 / 2}\left(1+\frac{1-4 \mu^{2}}{8} \frac{t}{x}\right)
$$

for every $x>1, t>0$.

Proof By the basic formula (10), with application of Eq. 12, we obtain

$$
\begin{aligned}
\frac{(2 \pi)^{1 / 2} t^{3 / 2}}{\lambda x^{\mu-1 / 2}} e^{\lambda^{2} / 2 t} q_{x}^{(-\mu)}(t) & =1+\frac{t}{x^{\mu-1 / 2}} \int_{0}^{\infty}\left(e^{-\kappa / 2 t}-1\right) w_{\lambda}(v) d v \\
& =1+\frac{1-4 \mu^{2}}{8} \frac{t}{x}+\frac{t}{x^{\mu-1 / 2}} \int_{0}^{\infty} e^{-\kappa / 2 t} w_{\lambda}(v) d v \\
& =1+\frac{1-4 \mu^{2}}{8} \frac{t}{x}+E(x, t) .
\end{aligned}
$$


Observe that, by Lemmas 14,16 and $17,\left|w_{\lambda}(v)\right| \leqslant C x^{\mu-3 / 2}$, which gives the following estimate

$$
\begin{aligned}
|E(x, t)| & =\left|\frac{t}{x^{\mu-1 / 2}} \int_{0}^{\infty} e^{-(\lambda v) / t} e^{-v^{2} / 2 t} w_{\lambda}(v) d v\right| \\
& \leqslant C \frac{t}{x} \int_{0}^{\infty} e^{-(\lambda v) / t} e^{-v^{2} / 2 t} d v \leqslant C \frac{t}{x}\left(\sqrt{t} \wedge \frac{t}{\lambda}\right) .
\end{aligned}
$$

This ends the proof of the first claim. For $\mu<1 / 2$ observe that

$$
0 \leqslant \int_{0}^{\infty}\left(e^{-\kappa / 2 t}-1\right) w_{\lambda}(v) d v \leqslant-\int_{0}^{\infty} w_{\lambda}(v) d v=x^{\mu-1 / 2}\left(1 / 4-\mu^{2}\right) / 2 x .
$$

Consequently, we get

$$
1 \leqslant \frac{(2 \pi)^{1 / 2} t^{3 / 2} x^{1 / 2}}{\lambda} e^{\lambda^{2} / 4 t} q_{x}^{(-\mu)}(t) \leqslant\left(1+\frac{1-4 \mu^{2}}{8} \frac{t}{x}\right)
$$

and this completes the proof of the second claim in the case $\mu<1 / 2$.

Remark 1 If $1<x<2$ then the absolute value of the error term is bounded by $C t^{3 / 2}$, while for $x>2$ it is bounded by $C\left(\frac{t}{x}\right)^{2}$. Observe also that the density $q_{x}^{(-\mu)}(t)$ up to a multiplicative constant is close to the density of the hitting distribution of 1 by the one-dimensional Brownian motion starting from $x$, when the fraction $\frac{t}{x}$ is small with the error precisely estimated by the above lemma. With some additional effort one can show that the error term estimate can not be improved.

Proposition 5 For every $\mu \neq 0$ and $c>0$ we have

$$
\lim _{x / t \rightarrow c, x \rightarrow \infty} \frac{1+x^{2 \mu}}{x^{|\mu|-1 / 2}} \frac{q_{x}^{(\mu)}(t) \sqrt{2 \pi t}}{e^{-\lambda^{2} / 2 t}}=\sqrt{\frac{\pi c}{2}} \frac{e^{-c}}{K_{|\mu|}(c)} .
$$

Proof It is enough to show the above-given convergence for strictly negative indices. The general statement follows from Eq. 8. Now we assume that $\mu>0$ and consider $q_{x}^{(-\mu)}(t)$. We define

$$
\begin{aligned}
w_{\infty}(v)= & -\sum_{i=1}^{k_{\mu}} \frac{\sqrt{z_{i}} e^{-z_{i}}}{K_{\mu-1}\left(z_{i}\right)} e^{-v z_{i}} \\
& -\frac{\cos (\pi \mu)}{\sqrt{2 \pi}} \int_{0}^{\infty} \frac{e^{u} K_{\mu}(u) e^{-v u} \sqrt{u} d u}{\cos ^{2}(\pi \mu) K_{\mu}^{2}(u)+\left(\pi I_{\mu}(u)+\sin (\pi \mu) K_{\mu}(u)\right)^{2}}
\end{aligned}
$$

for every $v>0$. If $k_{\mu}=0$ then the first sum is equal to zero. Using the asymptotic expansion (6) we easily see that

$$
\lim _{x \rightarrow \infty} \frac{\lambda}{x^{\mu-1 / 2}} w_{1, \lambda}(v)=-\sum_{i=1}^{k_{\mu}} \frac{\sqrt{z_{i}} e^{-z_{i}}}{K_{\mu-1}\left(z_{i}\right)} e^{-v z_{i}}
$$


The relation (5) implies that $\left|I_{\mu}(u)\right| \leqslant c_{2} \frac{e^{u}}{\sqrt{u}}$ and consequently

$$
\sqrt{x}\left|I \mu(x u) K_{\mu}(u)-I_{\mu}(u) K_{\mu}(x u)\right| e^{-x u} \leqslant c_{2} K_{\mu}(u) \frac{1}{\sqrt{u}}
$$

for every $u>0$. Moreover, using the estimates of $K_{\vartheta}$ and $I_{\vartheta}$ given in Section 2, we observe that the function

$$
f(u, v)=\frac{e^{u} K_{\mu}(u) e^{-v u} \sqrt{u} d u}{\cos ^{2}(\pi \mu) K_{\mu}^{2}(u)+\left(\pi I_{\mu}(u)+\sin (\pi \mu) K_{\mu}(u)\right)^{2}}
$$

is bounded, as a function of $u$, by $c_{3} e^{-(v+2) u} u^{3 / 2}$ on $[1, \infty)$ and by $c_{3} u^{\mu+1 / 2}$ on $(0,1)$ and consequently is integrable on $(0, \infty)$. Using the dominated convergence theorem we get

$$
\lim _{x \rightarrow \infty} \frac{\lambda}{x^{\mu-1 / 2}} w_{2, \lambda}(v)=-\frac{\cos (\pi \mu)}{\sqrt{2 \pi}} \int_{0}^{\infty} \frac{e^{u} K_{\mu}(u) e^{-v u} \sqrt{u} d u}{\cos ^{2}(\pi \mu) K_{\mu}^{2}(u)+\left(\pi I_{\mu}(u)+\sin (\pi \mu) K_{\mu}(u)\right)^{2}},
$$

which implies

$$
\lim _{x \rightarrow \infty} \frac{\lambda}{x^{\mu-1 / 2}} w_{\lambda}(v)=w_{\infty}(v), \quad v>0
$$

Using Eqs. 33 and 39 from Appendix we get that

$$
\begin{aligned}
\frac{\lambda}{x^{\mu-1 / 2}}\left|w_{\lambda}(v)\right| & \leqslant \frac{\lambda}{x^{\mu-1 / 2}}\left|w_{1, \lambda}(v)\right|+\frac{\lambda}{x^{\mu-1 / 2}}\left|w_{2, \lambda}(v)\right| \\
& \leqslant c_{4} e^{-v \theta_{\mu}}+c_{4} \frac{1}{(v+1)^{\mu+3 / 2}}
\end{aligned}
$$

for some positive $\theta_{\mu}$. Next, we take advantage of the following formula of the Laplace transform of $w_{\lambda}(v)$ (see Lemma 3.1 in [5])

$$
\frac{\lambda}{x^{\mu-1 / 2}} \int_{0}^{\infty} e^{-r v} w_{\lambda}(v) d v=\frac{r e^{\lambda r} x^{1 / 2} K_{\mu}(x r)}{K_{\mu}(r)}-\left(r-\left(\mu^{2}-1 / 4\right) \frac{\lambda}{2 x}\right), \quad r>0 .
$$

Thus, taking the limit as $x$ tends to $\infty$ in the above relation and applying the dominated convergence theorem we get

$$
\int_{0}^{\infty} e^{-r v} w_{\infty}(v) d v=\sqrt{\frac{\pi r}{2}} \frac{e^{-r}}{K_{\mu}(r)}-r+\frac{\mu^{2}-1 / 4}{2}
$$

for every $r>0$. Now let $c>0$. Observe that $\lim _{x / t \rightarrow c, x \rightarrow \infty} \frac{\kappa}{2 t}=c v$. Using Eq. 16 and the dominated convergence theorem we get

$$
\lim _{x / t \rightarrow c, x \rightarrow \infty} \frac{\lambda}{x^{\mu-1 / 2}} \int_{0}^{\infty} e^{-\kappa / 2 t} w_{\lambda}(v) d v=\int_{0}^{\infty} e^{-c v} w_{\infty}(v) d v .
$$


We have

$$
\frac{q_{x}^{(-\mu)}(t) \sqrt{2 \pi t}}{\lambda e^{-\lambda^{2} / 2 t}}=\frac{x^{\mu-1 / 2}}{t}-\left(\mu^{2}-1 / 4\right) \frac{x^{\mu-1 / 2}}{2 x}+\int_{0}^{\infty} e^{-\kappa / 2 t} w_{\lambda}(v) d v
$$

Multiplying both sides by $\frac{\lambda}{x^{\mu-1 / 2}}$, taking limit as $x / t \rightarrow c$ and $x \rightarrow \infty$ and using Eq. 17 we get

$$
\lim _{x / t \rightarrow c, x \rightarrow \infty} \frac{1}{x^{\mu-1 / 2}} \frac{q_{x}^{(-\mu)}(t) \sqrt{2 \pi t}}{e^{-\lambda^{2} / 2 t}}=c-\frac{\mu^{2}-1 / 4}{2}+\int_{0}^{\infty} e^{-c v} w_{\infty}(v) d v=\sqrt{\frac{\pi c}{2}} \frac{e^{-c}}{K_{\mu}(c)}>0 .
$$

Lemma 6 For every $C>0$ there is a constant $c_{1}>0$ depending on $C$ and $\mu>0$ such that

$$
\frac{1}{c_{1}} \lambda \frac{e^{-\lambda^{2} / 2 t}}{t^{3 / 2}} x^{\mu-1 / 2} \leqslant q_{x}^{(-\mu)}(t) \leqslant c_{1} \lambda \frac{e^{-\lambda^{2} / 2 t}}{t^{3 / 2}} x^{\mu-1 / 2},
$$

whenever $x<C t, x>1$.

Proof By Lemma 4 it is enough to consider $\mu>1 / 2$. Let $0<C^{\prime}<C$. The fact that the limit given in Proposition 5 exists and is strictly positive implies that for every $c \in\left[C^{\prime}, C\right]$ there exist $\varepsilon_{c}>0, D_{c}>1$ and $x_{c}>2$ such that

$$
\frac{1}{D_{c}} \leqslant \frac{1}{x^{\mu-1 / 2}} \frac{q_{x}^{(-\mu)}(t) \sqrt{2 \pi t}}{e^{-\lambda^{2} / 2 t}} \leqslant D_{c}
$$

for every $(x / t, x) \in\left(c-\varepsilon_{c}, c+\varepsilon_{c}\right) \times\left(x_{c}, \infty\right)$. The family

$$
\left\{\left(c-\varepsilon_{c}, c+\varepsilon_{c}\right)\right\}_{c \in\left[C^{\prime}, C\right]}
$$

is an open cover of the compact set $\left[C^{\prime}, C\right]$. Consequently, there exists a finite subcover $\left\{\left(c_{k}-\varepsilon_{c_{k}}, c_{k}+\varepsilon_{c_{k}}\right)\right\}_{k=1, \ldots, m}$. Setting $C^{*}=\max \left\{x_{c_{k}}: k=1, \ldots, m\right\}$ and $D=$ $\max \left\{D_{c_{k}}, k=1, \ldots, m\right\}$ we get

$$
\frac{1}{D} \leqslant \frac{1}{x^{\mu-1 / 2}} \frac{q_{x}^{(-\mu)}(t) \sqrt{2 \pi t}}{e^{-\lambda^{2} / 2 t}} \leqslant D
$$

for every $C^{\prime} \leqslant x / t \leqslant C$ and $x>C^{*}$, which proves the lemma for this range of $x$ and $t$. Choosing small enough the constant $C^{\prime}$ depending on $\mu$ we infer that using Lemma 4 we complete the proof in the case $x / t \leqslant C$ and $x>C^{*}$.

The estimates for $x \leqslant C^{*}$ and $C^{\prime} \leqslant x / t \leqslant C$ can be deduced from the absolute continuity property for Bessel processes with different indices. Indeed, from Eq. 7 we have

$$
x^{\mu-1 / 2} e^{-c_{\mu} t} q_{x}^{(-1 / 2)}(t) \leqslant q_{x}^{(-\mu)}(t) \leqslant x^{\mu-1 / 2} q_{x}^{(-1 / 2)}(t),
$$

where $c_{\mu}=\frac{\mu^{2}-1 / 4}{2}>0$. Moreover, we have

$$
q_{x}^{(-1 / 2)}(t)=\lambda \frac{e^{-\lambda^{2} / 2 t}}{\sqrt{2 \pi t^{3}}}
$$

and $\exp \left(-c_{\mu} t\right) \geqslant \exp \left(-c_{\mu} C^{*} / C^{\prime}\right)$. This ends the proof. 
In the next lemma we show a result which provides a satisfactory estimate when $t$ is large relative to $x$. This is done under some additional assumption on $\mu$.

Lemma 7 Suppose that $\mu-1 / 2 \in \mathbb{N}$. We have the following expansion

$$
q_{x}^{(-\mu)}(t)=\frac{\left(x^{2 \mu}-1\right)}{\Gamma(\mu) 2^{\mu}} e^{-\lambda^{2} / 2 t} \frac{1}{t^{\mu+1}}(1+E(x, t)) .
$$

There is a constant $c>0$ such that for $t>0$,

$$
|E(x, t)| \leqslant c \frac{x}{t} .
$$

Proof We use the following result proved in Lemma 4.4 of [5]. Let $l=\mu-1 / 2$. Then

$$
\begin{gathered}
\lim _{t \rightarrow \infty} t^{l+1} \int_{0}^{\infty} w_{\lambda}(v)\left(e^{-\kappa / 2 t}-\sum_{0 \leqslant j \leqslant l}(-1)^{j} \frac{1}{j !}\left(\frac{\kappa}{2 t}\right)^{j}\right) d v \\
=\frac{(-1)^{l+1}}{2^{l+1}(l+1) !} \int_{0}^{\infty} \kappa^{l+1} w_{\lambda}(v) d v=C_{l+1}(x)>0 .
\end{gathered}
$$

Let

$$
H(\lambda, t)=\int_{0}^{\infty} w_{\lambda}(v)\left(e^{-\kappa / 2 t}-\sum_{0 \leqslant j \leqslant l+1}(-1)^{j} \frac{1}{j !}\left(\frac{\kappa}{2 t}\right)^{j}\right) d v
$$

Using Eq. 19 we may write

$$
\begin{aligned}
\lambda \frac{e^{-\lambda^{2} / 2 t}}{\sqrt{2 \pi t}} H(\lambda, t)= & \lambda \frac{e^{-\lambda^{2} / 2 t}}{\sqrt{2 \pi t}} \int_{0}^{\infty} w_{\lambda}(v)\left(e^{-\kappa / 2 t}-\sum_{0 \leqslant j \leqslant l+1}(-1)^{j} \frac{1}{j !}\left(\frac{\kappa}{2 t}\right)^{j}\right) d v \\
= & \lambda \frac{e^{-\lambda^{2} / 2 t}}{\sqrt{2 \pi t}} \int_{0}^{\infty} w_{\lambda}(v)\left(e^{-\kappa / 2 t}-\sum_{0 \leqslant j \leqslant l}(-1)^{j} \frac{1}{j !}\left(\frac{\kappa}{2 t}\right)^{j}\right) d v \\
& -\lambda \frac{e^{-\lambda^{2} / 2 t}}{\sqrt{2 \pi t}} C_{l+1} t^{-l-1} \\
= & q_{x}^{(-\mu)}(t)-\lambda \frac{e^{-\lambda^{2} / 2 t}}{\sqrt{2 \pi t}} C_{l+1} t^{-l-1},
\end{aligned}
$$

where we applied Eq. 14 in the last step. Observe that

$$
\kappa^{l+2} \leqslant c\left(\lambda^{l+2} v^{l+2}+v^{2 l+4}\right),
$$

for some constant $c$. Next,

$$
\left|e^{-\kappa / 2 t}-\sum_{0 \leqslant j \leqslant l+1}(-1)^{j} \frac{1}{j !}\left(\frac{\kappa}{2 t}\right)^{j}\right| \leqslant\left(\frac{\kappa}{2 t}\right)^{l+2} \leqslant c\left(\frac{\lambda^{l+2} v^{l+2}+v^{2 l+4}}{t^{l+2}}\right),
$$


which together with the estimate (see Lemma 14 in Appendix)

$$
\left|w_{\lambda}(v)\right|=\left|w_{1, \lambda}(v)\right| \leqslant c x^{\mu-3 / 2} e^{-\theta_{\mu} v}
$$

leads to the following bound for $H(\lambda, t)$ :

$$
|H(\lambda, t)| \leqslant \int_{0}^{\infty}\left(\frac{\kappa}{2 t}\right)^{l+2}\left|w_{\lambda}(v)\right| d v \leqslant c x^{\mu-3 / 2} \frac{\left(\lambda^{l+2}+1\right)}{t^{l+2}} \approx \frac{x^{2 \mu}}{t^{l+2}} .
$$

To complete the proof we need to find the constant $C_{l+1}$. Let $T_{0}^{(-\mu)}$ denote the hitting time of 0 if we start the process from $x$. Due to the strong Markov property and the scaling property we obtain the following equality of the distributions:

$$
T_{0}^{(-\mu)} \stackrel{d}{=} \frac{1}{x^{2}} T_{0}^{(-\mu)}+T_{1}^{(-\mu)}
$$

where $T_{0}^{(-\mu)}$ and $T_{1}^{(-\mu)}$ are independent. It follows that

$$
P_{x}^{(-\mu)}\left(T_{0}^{(-\mu)}>t\right) \cong P_{x}^{(-\mu)}\left(T_{0}^{(-\mu)}>x^{2} t\right)+P_{x}^{(-\mu)}\left(T_{1}^{(-\mu)}>t\right), t \rightarrow \infty .
$$

Note that by the result of Getoor and Sharpe [9] we know that $t^{\mu} P_{x}^{(-\mu)}\left(T_{0}^{(-\mu)}>\right.$ $t) \cong \frac{x^{2 \mu}}{\Gamma(\mu+1) 2^{\mu}}$, which implies that

$$
t^{\mu} P_{x}^{(-\mu)}\left(T_{1}^{(-\mu)}>t\right) \cong \frac{x^{2 \mu}-1}{\Gamma(\mu+1) 2^{\mu}} .
$$

From Eq. 20 and

$$
q_{x}^{(-\mu)}(t)=\lambda \frac{e^{-\lambda^{2} / 2 t}}{\sqrt{2 \pi t}} C_{l+1} t^{-l-1}+\lambda \frac{e^{-\lambda^{2} / 2 t}}{\sqrt{2 \pi t}} H(\lambda, t)
$$

we infer

$$
t^{\mu} P_{x}^{(-\mu)}\left(T_{1}^{(-\mu)}>t\right) \cong \frac{\lambda}{\mu \sqrt{2 \pi}} C_{l+1}
$$

This in turn shows that $C_{l+1}(x)=\frac{\sqrt{2 \pi} \mu\left(x^{2 \mu}-1\right)}{\lambda \Gamma(\mu+1) 2^{\mu}}=\frac{\sqrt{2 \pi}\left(x^{2 \mu}-1\right)}{\lambda \Gamma(\mu) 2^{\mu}}$ and completes the proof.

Remark 2 Let $l=\mu-1 / 2 \in \mathbb{N}$ and $k \in \mathbb{N}$. Since all moments of $\kappa$ with respect to $w_{\lambda}(v) d v$ exist we can write

$$
\left|q_{x}^{(-\mu)}(t)-\lambda \frac{e^{-\lambda^{2} / 2 t}}{\sqrt{2 \pi t}} \sum_{i=1}^{k} \frac{C_{l+i}}{t^{l+i}}\right| \leqslant c \lambda \frac{e^{-\lambda^{2} / 2 t}}{\sqrt{2 \pi t}} \frac{x^{2 \mu+k-1}}{t^{l+k+1}},
$$

for some constant $c$, depending only on $\mu$ and $k$, where the constants $C_{l+i}=C_{l+i}(x)$ can be found by similar considerations as above. 
Lemma 8 Let $\mu-1 / 2 \notin \mathbb{N}$ and let $l=[\mu+1 / 2]$. There are constants $c_{1}, c_{2}, c_{3}$ depending only on $\mu$ such that

$$
c_{2} \frac{\lambda x^{2 \mu-1}}{t^{\mu+1}} e^{-\lambda^{2} / 2 t}\left(1-c_{3}\left(\frac{x}{t}\right)^{l-\mu+1 / 2}\right) \leqslant q_{x}^{(-\mu)}(t) \leqslant c_{1} \frac{\lambda x^{2 \mu-1}}{t^{\mu+1}} e^{-\lambda^{2} / 2 t},
$$

for $t>x>1$. Note that $l-\mu+1 / 2>0$.

Proof Applying Eq. 14 we have

$$
\begin{aligned}
q_{x}^{(-\mu)}(t)= & \lambda \frac{e^{-\lambda^{2} / 2 t}}{\sqrt{2 \pi t}} \int_{0}^{\infty}\left(e^{-\kappa / 2 t}-\sum_{0 \leqslant j \leqslant l}(-1)^{j} \frac{1}{j !}\left(\frac{\kappa}{2 t}\right)^{j}\right) w_{\lambda}(v) d v \\
= & \lambda \frac{e^{-\lambda^{2} / 2 t}}{\sqrt{2 \pi t}} \int_{0}^{\infty}\left(e^{-\kappa / 2 t}-\sum_{0 \leqslant j \leqslant l}(-1)^{j} \frac{1}{j !}\left(\frac{\kappa}{2 t}\right)^{j}\right) w_{\lambda, 1}(v) d v \\
& +\lambda \frac{e^{-\lambda^{2} / 2 t}}{\sqrt{2 \pi t}} \int_{0}^{\infty}\left(e^{-\kappa / 2 t}-\sum_{0 \leqslant j \leqslant l}(-1)^{j} \frac{1}{j !}\left(\frac{\kappa}{2 t}\right)^{j}\right) w_{\lambda, 2}(v) d v \\
= & q_{x, 1}^{(-\mu)}(t)+q_{x, 2}^{(-\mu)}(t) .
\end{aligned}
$$

The upper estimate of $q_{x, 1}^{(-\mu)}(t)$ is obtained using almost the same arguments as in the proof of Eq. 20 in Lemma 7. The resulting bound is of the following form:

$$
\left|q_{x, 1}^{(-\mu)}(t)\right| \leqslant c x^{2 \mu-1} \lambda \frac{e^{-\lambda^{2} / 2 t}}{t^{\mu+1}}\left(\frac{x}{t}\right)^{l-\mu+1 / 2} .
$$

Next, we deal with $q_{x, 2}^{(-\mu)}(t)$. Observing that

$$
e^{-\kappa / 2 t}-\sum_{0 \leqslant j \leqslant l}(-1)^{j} \frac{1}{j !}\left(\frac{\kappa}{2 t}\right)^{j} \approx(-1)^{l+1} \frac{\kappa^{l+1}}{t^{l}(\kappa+t)}
$$

and using Lemma 16 from Appendix, where the estimate of $w_{\lambda, 2}(v)$ is provided, we have

$$
q_{x, 2}^{(-\mu)}(t) \approx \lambda \frac{e^{-\lambda^{2} / 2 t}}{t^{l+1 / 2}} \int_{0}^{\infty} \frac{x^{2 \mu-1}}{(v+1)^{\mu+3 / 2}(v+x)^{\mu+1 / 2}} \frac{\kappa^{l+1}}{(\kappa+t)} d v
$$

We need to effectively estimate the integral

$$
J(t, x)=\int_{0}^{\infty} \frac{x^{2 \mu-1}}{(v+1)^{\mu+3 / 2}(v+x)^{\mu+1 / 2}} \frac{\kappa^{l+1}}{(\kappa+t)} d v .
$$

Using the following change of variables $\frac{\kappa}{t}=s$ we obtain $\frac{\kappa+v^{2}}{v t} d v=d s$, which yields

$$
\frac{d v}{v} \leqslant \frac{d s}{s} \leqslant 2 \frac{d v}{v}
$$


Assume that $x>2$. Thus, $\kappa=2 \lambda v+v^{2} \approx v(v+x)$ and

$$
J(t, x)=\int_{0}^{\infty} \frac{x^{2 \mu-1}(v+x)^{l-\mu+1 / 2} v^{l+1}}{(v+1)^{\mu+3 / 2}} \frac{1}{(\kappa+t)} d v=J_{1}(t, x)+J_{2}(t, x),
$$

where

$$
J_{1}(t, x)=\int_{0}^{1} \frac{x^{2 \mu-1}(v+x)^{l-\mu+1 / 2} v^{l+1}}{(v+1)^{\mu+3 / 2}} \frac{1}{(\kappa+t)} d v \approx x^{\mu+l-1 / 2} \int_{0}^{1} \frac{v^{l+1}}{(\kappa+t)} d v
$$

and

$$
J_{2}(t, x)=\int_{1}^{\infty} \frac{x^{2 \mu-1}(v+x)^{l-\mu+1 / 2} v^{l+1}}{(v+1)^{\mu+3 / 2}} \frac{1}{(\kappa+t)} d v \approx x^{2 \mu-1} \int_{1}^{\infty} \frac{\kappa^{l-\mu+1 / 2}}{v(\kappa+t)} d v .
$$

Applying the above change of variables and Eq. 22 we obtain

$$
\begin{aligned}
J_{2}(t, x) & \approx x^{2 \mu-1} \int_{1}^{\infty} \frac{\kappa^{l-\mu+1 / 2}}{v(\kappa+t)} d v \approx x^{2 \mu-1} \int_{(1+x) / t}^{\infty} \frac{(s t)^{l-\mu+1 / 2}}{s(s+1) t} d s \\
& =x^{2 \mu-1} \frac{t^{l}}{t^{\mu+1 / 2}} \int_{(1+x) / t}^{\infty} \frac{s^{l-\mu-1 / 2}}{s+1} d s .
\end{aligned}
$$

Observing that $\int_{0}^{\infty} \frac{s^{l-\mu-1 / 2}}{s+1} d s<\infty$ we arrive at

$$
J_{2}(t, x) \approx x^{2 \mu-1} \frac{t^{l}}{t^{\mu+1 / 2}}, \quad t>x .
$$

The first integral $J_{1}(t, x)$ we trivially estimate

$$
J_{1}(t, x) \leqslant c x^{2 \mu-l}\left(\frac{x}{t}\right)^{l+1 / 2-\mu} t^{l-1 / 2-\mu} \leqslant c J_{2}(t, x) .
$$

This yields the following estimate

$$
J(t, x) \approx x^{2 \mu-1} \frac{t^{l}}{t^{\mu+1 / 2}}, \quad t>x .
$$

Next, assume that $x \leqslant 2$. Thus, $\kappa \approx v^{2}, v \geqslant 1$, and

$$
J(t, x) \approx \int_{0}^{\infty} \frac{1}{(v+1)^{2 \mu+2}} \frac{(\lambda v)^{l+1}+v^{2(l+1)}}{(\kappa+t)} d v=J_{3}(t, x)+J_{4}(t, x),
$$

where

$$
J_{4}(t, x)=\int_{1}^{\infty} \frac{1}{(v+1)^{2 \mu+2}} \frac{(\lambda v)^{l+1}+v^{2(l+1)}}{(\kappa+t)} d v \approx \int_{1}^{\infty} \frac{v^{2(l-\mu)}}{\left(v^{2}+t\right)} d v \approx \frac{t^{l}}{t^{\mu+1 / 2}}
$$


Clearly

$$
J_{3}(t, x)=\int_{0}^{1} \frac{1}{(v+1)^{2 \mu+2}} \frac{(\lambda v)^{l+1}+v^{2(l+1)}}{(\kappa+t)} d v \leqslant c \frac{1}{t} \leqslant c J_{4}(t, x)
$$

if $t>1$. Obviously this implies that Eq. 23 holds in the case $x \leqslant 2$.

Using this estimate we finally obtain that

$$
\begin{aligned}
q_{x, 2}^{(-\mu)}(t) & \approx \lambda \frac{e^{-\lambda^{2} / 2 t}}{t^{l+1 / 2}} J(t, x) \\
& \approx \lambda x^{2 \mu-1} \frac{e^{-\lambda^{2} / 2 t}}{t^{\mu+1}}, \quad t>x>1 .
\end{aligned}
$$

A combination of Eqs. 21 and 24 ends the proof.

Lemma 9 Let $\mu=0$. For $t>2 x$,

$$
q_{x}^{(0)}(t) \approx \frac{\lambda}{x} \frac{e^{-\lambda^{2} / 2 t}}{t} \frac{1+\log x}{\left(1+\log \frac{t}{x}\right)(1+\log t)} .
$$

Proof Recalling the representation (10) for $q_{x}^{(-\mu)}(t)$ and observing that $e^{-\kappa / 2 t}-1 \approx$ $\frac{-\kappa}{(\kappa+t)}$ we have (note that $w_{\lambda}(v) \leqslant 0$ )

$$
\begin{aligned}
q_{x}^{(0)}(t) & =\lambda \frac{e^{-\lambda^{2} / 2 t}}{\sqrt{2 \pi t}}\left(x^{-1 / 2} / 2 t+\int_{0}^{\infty}\left(e^{-\kappa / 2 t}-1\right) w_{\lambda}(v) d v\right) \\
& \approx \lambda \frac{e^{-\lambda^{2} / 2 t}}{\sqrt{2 \pi t}}\left(x^{-1 / 2} / 2 t+\int_{0}^{\infty} \frac{\kappa}{(\kappa+t)}\left(-w_{\lambda}(v)\right) d v\right) .
\end{aligned}
$$

Hence, it is enough to estimate the integral

$$
J(t, x)=\int_{0}^{\infty} \frac{\kappa}{(\kappa+t)}\left(-w_{\lambda}(v)\right) d v .
$$

We start with the case $x>2$. Due to Lemma 17 (see Appendix) we have

$$
-w_{\lambda}(v) \approx \begin{cases}\frac{1}{x^{3 / 2}}, & v<3 / 2, \\ \frac{1}{x^{3 / 2} v^{3 / 2} \log v}, & 3 / 2 \leqslant v<x, \\ \frac{\log x}{x v^{2} \log ^{2} v}, & v \geqslant x>2 .\end{cases}
$$

We write

$$
\begin{aligned}
J(t, x) & =\left(\int_{0}^{2}+\int_{2}^{x}+\int_{x}^{\infty}\right) \frac{\kappa}{(\kappa+t)}\left(-w_{\lambda}(v)\right) d v \\
& =J_{1}(t, x)+J_{2}(t, x)+J_{3}(t, x) .
\end{aligned}
$$


Note that

$$
\frac{\kappa}{(\kappa+t)} \approx\left\{\begin{array}{l}
\frac{v x}{(v x+t)}, v<x, \\
\frac{v^{2}}{\left(v^{2}+t\right)}, v \geqslant x,
\end{array}\right.
$$

which implies

$$
\begin{aligned}
J_{1}(t, x) & \approx \int_{0}^{3 / 2} \frac{1}{x^{3 / 2}} \frac{\kappa}{(\kappa+t)} d v \approx \int_{0}^{3 / 2} \frac{1}{x^{3 / 2}} \frac{v x}{(v x+t)} d v \leqslant \frac{3}{2 x^{1 / 2} t} \\
J_{2}(t, x) & \approx \int_{3 / 2}^{x} \frac{1}{x^{3 / 2} v^{3 / 2} \log v} \frac{\kappa}{(\kappa+t)} d v \approx \int_{3 / 2}^{x} \frac{1}{x^{3 / 2} v^{3 / 2} \log v} \frac{v x}{(v x+t)} d v, \\
J_{3}(t, x)(t, x) & \approx \int_{x}^{\infty} \frac{\log x}{x v^{2} \log ^{2} v} \frac{\kappa}{(\kappa+t)} d v \approx \int_{x}^{\infty} \frac{\log x}{x v^{2} \log ^{2} v} \frac{v^{2}}{\left(v^{2}+t\right)} d v .
\end{aligned}
$$

Assume that $2 x<t<x^{2}$. First, we deal with

$$
\begin{aligned}
J_{2}(t, x) & \approx \frac{1}{x^{1 / 2}} \int_{3 / 2}^{t / x} \frac{1}{v^{1 / 2} \log v} \frac{1}{(v x+t)} d v+\frac{1}{x^{1 / 2}} \int_{t / x}^{x} \frac{1}{v^{1 / 2} \log v} \frac{1}{(v x+t)} d v \\
& \approx \frac{1}{x^{1 / 2} t} \int_{3 / 2}^{t / x} \frac{1}{v^{1 / 2} \log v} d v+\frac{1}{x^{3 / 2}} \int_{t / x}^{x} \frac{1}{v^{3 / 2} \log v} d v \\
& \approx \frac{1}{x^{1 / 2} t} \int_{3 / 2}^{t / x} \frac{1}{v^{1 / 2} \log v} d v+\frac{1}{x^{3 / 2}} \int_{t / x}^{x} \frac{1}{v^{3 / 2} \log v} d v .
\end{aligned}
$$

We have

$$
\int_{3 / 2}^{t / x} \frac{1}{v^{1 / 2} \log v} d v \approx \sqrt{t / x} \frac{1}{\log \frac{t}{x}}
$$

and

$$
\int_{t / x}^{x} \frac{1}{v^{3 / 2} \log v} d v \leqslant 2 \sqrt{x / t} \frac{1}{\log \frac{t}{x}}
$$

which shows that

$$
J_{2}(t, x) \approx \frac{1}{x \sqrt{t}} \frac{1}{\log \frac{t}{x}} .
$$

Next,

$$
J_{3}(t, x)(t, x) \approx \int_{x}^{\infty} \frac{\log x}{x \log ^{2} v} \frac{1}{\left(v^{2}+t\right)} d v \approx \frac{\log x}{x} \int_{x}^{\infty} \frac{1}{v^{2} \log ^{2} v} d v \approx \frac{1}{x^{2} \log x} .
$$


Combining all the estimates we see that for $2 x<t<x^{2}$ we have

$$
J(t, x) \approx J_{2}(t, x) \approx \frac{1}{x \sqrt{t}} \frac{1}{\log \frac{t}{x}} .
$$

Next, we assume that $t>x^{2}$.

$$
\begin{aligned}
J_{3}(t, x)(t, x) & \approx \int_{x}^{\sqrt{t}} \frac{\log x}{x \log ^{2} v} \frac{1}{\left(v^{2}+t\right)} d v+\int_{\sqrt{t}}^{\infty} \frac{\log x}{x \log ^{2} v} \frac{1}{\left(v^{2}+t\right)} d v \\
& \approx \frac{\log x}{x t} \int_{x}^{\sqrt{t}} \frac{1}{\log ^{2} v} d v+\frac{\log x}{x} \int_{\sqrt{t}}^{\infty} \frac{1}{v^{2} \log ^{2} v} d v .
\end{aligned}
$$

Observe that

$$
\int_{x}^{\sqrt{t}} \frac{1}{\log ^{2} v} d v \leqslant \int_{2}^{\sqrt{t}} \frac{1}{\log ^{2} v} d v \approx \frac{\sqrt{t}}{\log ^{2} t}
$$

and

$$
\int_{\sqrt{t}}^{\infty} \frac{1}{v^{2} \log ^{2} v} d v \approx \frac{1}{\sqrt{t} \log ^{2} t}
$$

As a consequence we obtain

$$
J_{3}(t, x)(t, x) \approx \frac{1}{x \sqrt{t}} \frac{\log x}{\log ^{2} t} .
$$

Next,

$$
\begin{aligned}
J_{2}(t, x) & \approx \frac{1}{x^{1 / 2}} \int_{3 / 2}^{x} \frac{1}{v^{1 / 2} \log v} \frac{1}{(v x+t)} d v \\
& \approx \frac{1}{x^{1 / 2} t} \int_{3 / 2}^{x} \frac{1}{v^{1 / 2} \log v} d v \\
& \approx \frac{1}{t \log x} \leqslant C \frac{1}{x \sqrt{t}} \frac{\log x}{\log ^{2} t}, x^{2} \leqslant t .
\end{aligned}
$$

Recall that

$$
J_{1}(t, x) \leqslant \frac{3}{2 x^{1 / 2} t}
$$

Hence, in this case, it is easily seen that the integral $J_{3}(t, x)$ dominates and

$$
J(t, x) \approx J_{3}(t, x)(t, x) \approx \frac{1}{x \sqrt{t}} \frac{\log x}{\log ^{2} t}, x^{2} \leqslant t .
$$


Summarizing all the estimates obtained for $J(t, x)$ we have that for $4<2 x<t<x^{2}$,

$$
\begin{aligned}
q_{x}^{(0)}(t) & =\lambda \frac{e^{-\lambda^{2} / 2 t}}{\sqrt{2 \pi t}}\left(x^{-1 / 2} / 2 t+\int_{0}^{\infty}\left(e^{-\kappa / 2 t}-1\right) w_{\lambda}(v) d v\right) \\
& \approx \lambda \frac{e^{-\lambda^{2} / 2 t}}{\sqrt{2 \pi t}}\left(x^{-1 / 2} / 2 t+\frac{1}{x \sqrt{t}} \frac{1}{\log \frac{t}{x}}\right) \\
& \approx \frac{e^{-\lambda^{2} / 2 t}}{t} \frac{1}{\log \frac{t}{x}} \\
& \approx \frac{\lambda}{x} \frac{e^{-\lambda^{2} / 2 t}}{t} \frac{1+\log x}{\left(1+\log \frac{t}{x}\right)(1+\log t)},
\end{aligned}
$$

while for $t>x^{2}$,

$$
\begin{aligned}
q_{x}^{(0)}(t) & =\lambda \frac{e^{-\lambda^{2} / 2 t}}{\sqrt{2 \pi t}}\left(x^{-1 / 2} / 2 t+\int_{0}^{\infty}\left(e^{-\kappa / 2 t}-1\right) w_{\lambda}(v) d v\right) \\
& \approx \lambda \frac{e^{-\lambda^{2} / 2 t}}{\sqrt{2 \pi t}}\left(x^{-1 / 2} / 2 t+\frac{1}{x \sqrt{t}} \frac{\log x}{\log ^{2} t}\right) \\
& \approx \frac{e^{-\lambda^{2} / 2 t}}{t} \frac{\log x}{\log ^{2} t} \\
& \approx \frac{\lambda}{x} \frac{e^{-\lambda^{2} / 2 t}}{t} \frac{1+\log x}{\left(1+\log \frac{t}{x}\right)(1+\log t)} .
\end{aligned}
$$

This completes the proof in the case $x>2$.

Finally, assume that $1<x \leqslant 2 \leqslant t$. By Lemma 17 (see Appendix) we have

$$
-w_{\lambda}(v) \approx \begin{cases}1, & v \leqslant 2 \\ \frac{1}{v^{2}\left(\log ^{2} v+1\right)}, & v>2 .\end{cases}
$$

Thus, using the fact that for such range of $x$ we have

$$
1-e^{-\kappa / 2 t} \approx \frac{\kappa}{\kappa+t} \approx \frac{v^{2}}{v^{2}+t}
$$

we obtain

$$
q_{x}^{(0)}(t) \approx \lambda \frac{e^{-\lambda^{2} / 2 t}}{\sqrt{t}}\left(\frac{1}{x^{1 / 2} t}+\int_{0}^{2} \frac{v^{2}}{v^{2}+t} d v+\int_{2}^{\infty} \frac{d v}{\left(v^{2}+t\right)\left(\log ^{2} v+1\right)}\right) .
$$

Obviously, we have

$$
\int_{0}^{2} \frac{v^{2}}{v^{2}+t} d v \approx \frac{1}{t}
$$

and

$$
\int_{2}^{\infty} \frac{d v}{\left(v^{2}+t\right)\left(\log ^{2} v+1\right)}=\frac{1}{\sqrt{t}} \int_{\frac{2}{\sqrt{t}}}^{\infty} \frac{1}{\left(s^{2}+1\right)} \frac{d s}{(\log (s)+\log \sqrt{t})^{2}+1} \approx \frac{1}{\sqrt{t} \log ^{2} t}
$$


Hence, for $1<x \leqslant 2 \leqslant t$, we have

$$
\begin{aligned}
q_{x}^{(0)}(t) & \approx \lambda \frac{e^{-\lambda / 4 t}}{t \log ^{2} t} \approx \lambda \frac{e^{-\lambda / 4 t}}{t} \frac{1+\log x}{\log ^{2} t} \\
& \approx \frac{\lambda}{x} \frac{e^{-\lambda^{2} / 2 t}}{t} \frac{1+\log x}{\left(1+\log \frac{t}{x}\right)(1+\log t)} .
\end{aligned}
$$

The proof is completed.

\section{Applications}

\subsection{Survival Probabilities of Killed Bessel Process}

In this subsection we introduce uniform estimates for the survival probabilities of Bessel process killed when exiting the half-line $(1, \infty)$. The theorem below is formulated for processes with non-negative indices, however due to Eq. 8 we can easily derive the corresponding result for strictly negative indices.

Theorem 10 Let $\mu>0$. Then, for every $t \geqslant 0$ and $x>1$, we have

$$
\boldsymbol{P}_{x}^{(\mu)}\left(t<T_{1}^{(\mu)}<\infty\right) \approx \frac{x-1}{\sqrt{x \wedge t}+x-1} \frac{1}{t^{\mu}+x^{2 \mu}} .
$$

Moreover, for every $t \geqslant 0$ and $x>1$, we have

$$
\boldsymbol{P}_{x}^{(0)}\left(T_{1}^{(0)}>t\right) \approx 1 \wedge \frac{\log x}{\log \left(1+t^{1 / 2}\right)} .
$$

Proof Using Eq. 15 we get

$$
\begin{aligned}
\mathbf{P}_{x}^{(\mu)} & \left(t<T_{1}^{(\mu)}<\infty\right) \\
& =\int_{t}^{\infty} q_{x}^{(\mu)}(s) d s \\
& \approx \frac{(x-1)}{x^{2 \mu}}\left(x^{\mu-1 / 2} \int_{t}^{t \vee x} s^{-3 / 2} e^{-(x-1)^{2} / 2 s} d s+x^{2 \mu-1} \int_{t \vee x}^{\infty} s^{-\mu-1} e^{-(x-1)^{2} / 2 s} d s\right) \\
& =J_{1}(t, x)+J_{2}(t, x) .
\end{aligned}
$$

The integral $J_{2}(t, x)$ can be estimated as follows

$$
\begin{aligned}
J_{2}(t, x) & =\frac{x-1}{x} \int_{t \vee x}^{\infty} s^{-\mu-1} e^{-(x-1)^{2} / 2 s} d s=\frac{(x-1)^{1-2 \mu}}{x} \int_{0}^{\frac{(x-1)^{2}}{2(t \vee x)}} u^{\mu-1} e^{-u} d u \\
& \approx \frac{(x-1)^{1-2 \mu}}{x}\left(\frac{(x-1)^{2 \mu}}{(t \vee x)^{\mu}} \wedge 1\right)=\frac{x-1}{x}\left(\frac{1}{(t \vee x)^{\mu}} \wedge \frac{1}{(x-1)^{2 \mu}}\right) \\
& \approx \frac{x-1}{x} \frac{1}{(t \vee x)^{\mu}+(x-1)^{2 \mu}} .
\end{aligned}
$$


Observe also that for $t \geqslant x$ we have

$$
\begin{aligned}
\frac{1}{(t \vee x)^{\mu}+(x-1)^{2 \mu}} & =\frac{1}{t^{\mu}+(x-1)^{2 \mu}} \approx \frac{1}{t^{\mu}+x^{2 \mu}}, \\
\frac{x-1}{x} & \approx \frac{x-1}{\sqrt{x}-1+x}=\frac{x-1}{\sqrt{x \wedge t}+x-1} .
\end{aligned}
$$

The fact that for $t \geqslant x$ the integral $J_{1}(t, x)$ vanishes together with the above-given estimates of $J_{2}(t, x)$ end the proof in that case. If $t<x$, then

$$
J_{2}(t, x) \approx \frac{x-1}{x} \frac{1}{x^{\mu}+(x-1)^{2 \mu}} \approx \frac{x-1}{x} \frac{1}{t^{\mu}+x^{2 \mu}} .
$$

Substituting $u=(x-1)^{2} /(2 t)$ we can rewrite $J_{1}(t, x)$ in the following way

$$
J_{1}(t, x)=\frac{x-1}{x^{\mu+1 / 2}} \int_{t}^{x} s^{-3 / 2} e^{-(x-1)^{2} / 2 s} d s=\frac{\sqrt{2}}{x^{\mu+1 / 2}} \int_{\frac{(x-1)^{2}}{2 x}}^{\frac{(x-1)^{2}}{2 t}} u^{-1 / 2} e^{-u} d u, \quad t<x .
$$

For $x \geqslant 2$ we have

$$
J_{1}(t, x) \leqslant \frac{1}{2^{\mu}} \int_{\frac{(x-1)^{2}}{2 x}}^{\infty} u^{-1 / 2} e^{-u} d u \approx\left(\frac{(x-1)^{2}}{2 x}\right)^{1 / 2} \exp \left(-\frac{(x-1)^{2}}{2 x}\right)
$$

and it means that $J_{1}(t, x)$ is dominated by $J_{2}(t, x)$ in that region. Consequently, we obtain

$$
\mathbf{P}_{x}^{(\mu)}\left(t<T_{1}^{(\mu)}<\infty\right) \approx J_{2}(t, x) \approx \frac{x-1}{x} \frac{1}{t^{\mu}+x^{2 \mu}} \approx \frac{x-1}{\sqrt{t}+x-1} \frac{1}{t^{\mu}+x^{2 \mu}}
$$

whenever $t<x$ and $x \geqslant 2$. Moreover, for $1<x<2, t<x$ and $(x-1)>\sqrt{t}$ we get

$$
\frac{1}{2^{\mu}} \int_{1 / 4}^{1 / 2} u^{-1 / 2} e^{-u} d u \leqslant J_{1}(t, x) \leqslant \int_{0}^{\infty} u^{-1 / 2} e^{-u} d u .
$$

Thus, the integral $J_{1}(t, x)$ dominates $J_{2}(t, x)$ and we have

$$
\mathbf{P}_{x}^{(\mu)}\left(t<T_{1}^{(\mu)}<\infty\right) \approx J_{1}(t, x) \approx 1 \approx \frac{x-1}{\sqrt{t}+x-1} \frac{1}{t^{\mu}+u^{2 \mu}} .
$$

Finally, for $1<x<2, t<x$ and $(x-1) \leqslant \sqrt{t}$ we get

$$
J_{1}(t, x) \approx \int_{\frac{(x-1)^{2}}{2 x}}^{\frac{(x-1)^{2}}{2 t}} u^{-1 / 2} d u=\sqrt{2}(x-1)\left(\frac{1}{\sqrt{t}}-\frac{1}{\sqrt{x}}\right), \quad J_{2}(t, x) \approx x-1 .
$$

Thus we get $1-1 / \sqrt{x} \leqslant(\sqrt{x}-1) / \sqrt{t} \leqslant \sqrt{2} / \sqrt{t}$ and consequently

$$
\mathbf{P}_{x}^{(\mu)}\left(t<T_{1}^{(\mu)}<\infty\right) \approx(x-1)\left(\frac{1}{\sqrt{t}}-\frac{1}{\sqrt{x}}+1\right) \approx \frac{x-1}{\sqrt{t}} \approx \frac{x-1}{\sqrt{t}+x-1} \frac{1}{t^{\mu}+x^{2 \mu}} .
$$


Now we deal with the case $\mu=0$. We begin with the case of large time $t \geqslant 2$. We have to consider three cases. For $s \geqslant t \geqslant x^{2}$ we have

$$
q_{x}^{(0)}(s) \approx \frac{\lambda}{x s} \frac{1+\log x}{\log ^{2} s} .
$$

Consequently

$$
\mathbf{P}_{x}^{(0)}\left(T_{1}^{(0)}>t\right) \approx \frac{x-1}{x} \int_{t}^{\infty} \frac{1+\log x}{s \log ^{2} s} d s \approx \frac{(x-1)(1+\log x)}{x \log t} \approx \frac{\log x}{\log \left(1+t^{1 / 2}\right)} .
$$

If $2 \leqslant t \leqslant x^{2}$ and additionally $x \geqslant 2$, using the above estimate, we have

$$
1 \geqslant \mathbf{P}_{x}^{(0)}\left(T_{1}^{(0)}>t\right) \geqslant \mathbf{P}_{x}^{(0)}\left(T_{1}^{(0)}>x^{2}\right) \approx \frac{\log x}{\log (1+x)} \approx 1 .
$$

Finally, for $2 \leqslant t \leqslant x^{2}$ with $x<2$ we get $2<t<4$ and we can write

$$
\mathbf{P}_{x}^{(0)}\left(T_{1}^{(0)}>t\right)=\mathbf{P}_{x}^{(0)}\left(t<T_{1}^{(0)} \leqslant 10\right)+\mathbf{P}_{x}^{(0)}\left(T_{1}^{(0)} \geqslant 10\right) \approx \lambda .
$$

To justify the last approximation observe that, using Lemma 4, we get

$$
q_{x}^{(0)}(s) \approx \lambda, \quad 2 \leqslant t \leqslant s \leqslant 10,
$$

and it gives

$$
\mathbf{P}_{x}^{(0)}\left(t<T_{1}^{(0)} \leqslant 10\right)=\int_{t}^{10} q_{x}^{(0)}(s) d s \approx \lambda .
$$

Moreover, using Eq. 25 we also get

$$
\mathbf{P}_{x}^{(0)}\left(T_{1}^{(0)} \geqslant 10\right) \approx \lambda
$$

Combining all cases we obtain that

$$
\mathbf{P}_{x}^{(0)}\left(T_{1}^{(0)}>t\right) \approx 1 \wedge \frac{\log x}{\log \left(1+t^{1 / 2}\right)}, \quad t \geqslant 2 .
$$

In the case of small times $t \leqslant 2$ and $1<x<2$, by Lemma 4, we have

$$
q_{x}^{(0)}(s) \approx \lambda \frac{e^{-\lambda^{2} / 2 s}}{s^{3 / 2}}, \quad t \leqslant s \leqslant 10 .
$$

and thus

$$
\mathbf{P}_{x}^{(0)}\left(t<T_{1}^{(0)} \leqslant 10\right) \approx \int_{t}^{10} \lambda \frac{e^{-\lambda^{2} / 2 s}}{s^{3 / 2}} d s \approx 1 \wedge \frac{\lambda}{t^{1 / 2}}
$$

Observe also that $\mathbf{P}_{x}^{(0)}\left(T_{1}^{(0)} \geqslant 10\right) \approx \lambda$ by our previous estimates in the case of large times. Hence

$$
\mathbf{P}_{x}^{(0)}\left(T_{1}^{(0)}>t\right) \approx 1 \wedge \frac{\lambda}{t^{1 / 2}}
$$


Finally, for $t \leqslant 2$ and $x>2$, using the Markov property, one can easily obtain that

$$
\mathbf{P}_{x}^{(0)}\left(T_{1}^{(0)}>t\right) \geqslant \mathbf{P}_{2}^{(0)}\left(T_{1}^{(0)}>2\right) \approx 1
$$

Again combining all the cases we easily obtain

$$
\mathbf{P}_{x}^{(0)}\left(T_{1}^{(0)}>t\right) \approx 1 \wedge \frac{\lambda}{t^{1 / 2}} \approx 1 \wedge \frac{\log x}{\log \left(1+t^{1 / 2}\right)}, \quad t \leqslant 2 .
$$

This ends the proof.

\subsection{Poisson Kernel for Hyperbolic Brownian Motion with Drift}

Before providing the precise bounds of the Poisson kernel of half-spaces for hyperbolic Brownian motion with drift, we introduce some basic notation related to hyperbolic spaces and hyperbolic Brownian motion and its connections with Bessel processes (see [7] and [11] for more details).

Let us consider a half-space model of $n$-dimensional real hyperbolic space

$$
\mathbb{H}^{n}=\left\{\left(y_{1}, \ldots, y_{n-1}, y_{n}\right) \in \mathbb{R}^{n}: y_{n}>0\right\}
$$

with Riemannian metric

$$
d s^{2}=\frac{d y_{1}^{2}+\ldots+d y_{n-1}^{2}+d y_{n}^{2}}{y_{n}^{2}} .
$$

The hyperbolic distance $d_{\mathbb{H}^{n}}(y, z)$ is given by

$$
\cosh d_{\mathbb{H}^{n}}(y, z)=1+\frac{|y-z|^{2}}{2 y_{n} z_{n}}, \quad y, z \in \mathbb{H}^{n} .
$$

The Laplace-Beltrami operator associated with the metric is given by

$$
\Delta_{\mathbb{H}^{n}}=y_{n}^{2} \sum_{i=1}^{n} \frac{\partial^{2}}{\partial y_{i}^{2}}-(n-2) y_{n} \frac{\partial}{\partial y_{n}} .
$$

For every $\mu>0$, we also define the following operator

$$
\Delta_{\mu}=\Delta_{\mathbb{H}^{n}}-(2 \mu-n+1) y_{n} \frac{\partial}{\partial y_{n}}=y_{n}^{2} \sum_{i=1}^{n} \frac{\partial^{2}}{\partial y_{i}^{2}}-(2 \mu-1) y_{n} \frac{\partial}{\partial y_{n}} \text {. }
$$

The hyperbolic Brownian motion (HBM) with drift is a diffusion $Y^{(\mu)}=\left\{Y_{t}^{(\mu)}, t \geqslant 0\right\}$ on $\mathbb{H}^{n}$ with a generator $\frac{1}{2} \Delta_{\mu}$. For $\mu=\frac{n-1}{2}$ we obtain the standard HBM on $\mathbb{H}^{n}$ (with $\frac{1}{2} \Delta_{\mathbb{H}^{n}}$ as a generator).

The structure of the process $Y^{(\mu)}$ starting from $\left(\tilde{y}, y_{n}\right) \in \mathbb{H}^{n}$ can be described in terms of the geometric Brownian motion and integral functional $A_{x}^{(-\mu)}$ as follows. Let $\tilde{B}=\left\{\tilde{B}_{t}, t \geqslant 0\right\}$ be $(n-1)$-dimensional Brownian motion starting from $\tilde{y} \in \mathbb{R}^{n-1}$ 
independent from a geometric Brownian motion $X^{(-\mu)}$ starting from $y_{n}>0$. Then we have

$$
Y_{t}^{(\mu)} \stackrel{d}{=}\left(\tilde{B}\left(A_{y_{n}}^{(-\mu)}(t)\right), X_{t}^{(-\mu)}\right) .
$$

We consider $D=\left\{\left(y_{1}, \ldots, y_{n-1}, y_{n}\right) \in \mathbb{H}^{n}: y_{n}>1\right\}$ and the first exit time of $Y^{(\mu)}$ from $D$

$$
\tau_{D}=\inf \left\{t \geqslant 0: Y_{t}^{(\mu)} \notin D\right\}=\inf \left\{t \geqslant 0: X_{t}^{(-\mu)} \notin(1, \infty)\right\}=\tau,
$$

where $\tau=$ is the first exit time from the set $(1, \infty)$ of a geometric Brownian motion $X^{(-\mu)}$ defined in Section 2. We denote by $P^{(\mu)}(y, z), y \in D$ and $z \in \partial D$, the Poisson kernel of $D$, i.e. the density of the distribution of $Y_{\tau_{D}}^{\mu}$, with $Y_{0}^{(\mu)}=y_{n}>1$.

Theorem 11 For every $\mu>0$ we have

$$
P^{(\mu)}(y, z) \approx \frac{y_{n}-1}{|z-y|^{n}}\left(\frac{y_{n}}{\cosh d_{\mathbb{H}^{n}}(y, z)}\right)^{\mu-1 / 2},
$$

where $y=\left(\tilde{y}, y_{n}\right), y_{n}>1$ and $z=(\tilde{z}, 1), \tilde{z} \in \mathbb{R}^{n-1}$.

Proof Let us denote by

$$
g_{t}(w)=\frac{\exp \left(-|w|^{2} / 2 t\right)}{(2 \pi t)^{(n-1) / 2}}, \quad w \in \mathbb{R}^{n-1}
$$

the Brownian motion transition density in $\mathbb{R}^{n-1}, n=2,3, \ldots$ Using the fact that $\tilde{B}$ and $A_{y_{n}}^{(-\mu)}(\tau)$ are independent we obtain

$$
P^{(\mu)}(y, z)=\int_{0}^{\infty} g_{t}(\tilde{z}-\tilde{y}) q_{y_{n}}^{(-\mu)}(t) d t, \quad y_{n}>1, z \in \mathbb{R}^{n-1} .
$$

Using the estimates given in Eq. 15 we obtain

$$
\begin{aligned}
P^{(\mu)}(y, z) & \approx \lambda \int_{0}^{\infty} e^{-\left(|\tilde{z}-\tilde{y}|^{2}+\lambda^{2}\right) / 2 t} \frac{y_{n}^{2 \mu-1}}{t^{\mu-1 / 2}+y_{n}^{\mu-1 / 2}} \frac{d t}{t^{(n+2) / 2}} \\
& \approx \lambda\left(y_{n}^{\mu-1 / 2} \int_{0}^{y_{n}} e^{-\left(|\tilde{z}-\tilde{y}|^{2}+\lambda^{2}\right) / 2 t} \frac{d t}{t^{(n+2) / 2}}+y_{n}^{2 \mu-1} \int_{y_{n}}^{\infty} e^{-\left(|\tilde{z}-\tilde{y}|^{2}+\lambda^{2}\right) / 2 t} \frac{d t}{t^{(n+1) / 2+\mu}}\right) \\
& =\lambda y_{n}^{\mu-n / 2-1 / 2}\left[\rho^{-n / 2} \int_{\rho}^{\infty} u^{n / 2-1} e^{-u} d u+\rho^{1 / 2-\mu-n / 2} \int_{0}^{\rho} u^{n / 2-3 / 2+\mu} e^{-u} d u\right]
\end{aligned}
$$

where $\rho=\frac{|\tilde{z}-\tilde{y}|^{2}+\lambda^{2}}{2 y_{n}}, \lambda=y_{n}-1$. Using Eq. 28 (see Appendix) we can see that

$$
P^{(\mu)}(y, z) \approx \lambda \frac{y_{n}^{\mu-1 / 2}}{\left(|\tilde{z}-\tilde{y}|^{2}+\lambda^{2}\right)^{n / 2}}, \quad \frac{2 y_{n}}{|\tilde{z}-\tilde{y}|^{2}+\lambda^{2}} \geqslant 1
$$

and

$$
P^{(\mu)}(y, z) \approx \lambda \frac{y_{n}^{\mu-1 / 2}}{\left(|\tilde{z}-\tilde{y}|^{2}+\lambda^{2}\right)^{n / 2}} \frac{\left(2 y_{n}\right)^{\mu-1 / 2}}{\left(|\tilde{z}-\tilde{y}|^{2}+\lambda^{2}\right)^{\mu-1 / 2}}, \quad \frac{2 y_{n}}{|\tilde{z}-\tilde{y}|^{2}+\lambda^{2}}<1 .
$$


Combining both estimates and using the formula for the hyperbolic distance we obtain

$$
\begin{aligned}
P^{(\mu)}(y, z) & \approx \lambda \frac{y_{n}^{\mu-1 / 2}}{\left(|\tilde{z}-\tilde{y}|^{2}+\lambda^{2}\right)^{n / 2}}\left(\frac{1}{1+\frac{|\tilde{z}-\tilde{y}|^{2}+\lambda^{2}}{2 y_{n}}}\right)^{\mu-1 / 2} \\
& =\frac{\lambda}{|z-y|^{n}}\left(\frac{y_{n}}{\cosh d_{\mathbb{H}^{n}}(y, z)}\right)^{\mu-1 / 2} .
\end{aligned}
$$

Remark 3 The operator $\Delta_{\mu}$ is strongly elliptic operator on every bounded (in hyperbolic metric) subset of $\mathbb{H}^{n}$. Consequently, the hyperbolic Poisson kernels of such set are comparable with Euclidean ones. However, considered set $D$ is unbounded in $\mathbb{H}^{n}$ and the general comparison results can not be applied. Besides, the function $P^{(\mu)}(y, z)$ for $\mu \neq 1 / 2$ is no longer comparable with Euclidean Poisson kernel of upper half-space and the difference in behavior of those two functions is described by the factor

$$
\left(\frac{y_{n}}{\cosh d_{\mathbb{H}^{n}}(y, z)}\right)^{\mu-1 / 2} .
$$

Acknowledgement The authors are grateful to the referee for remarks and comments which enabled them to improve the presentation of the paper.

Open Access This article is distributed under the terms of the Creative Commons Attribution License which permits any use, distribution, and reproduction in any medium, provided the original author(s) and the source are credited.

\section{Appendix}

\section{A.1 Uniform Estimates for Some Class of Integrals}

Lemma 12 For $v \geqslant 0,0 \leqslant a<b$ and $d>0$ we have

$$
\int_{a}^{b} u^{v} e^{-d u} d u \stackrel{c}{\approx} b^{v}\left(\frac{a+\frac{1}{d}}{b+\frac{1}{d}}\right)^{v} e^{-a d} \frac{b-a}{d(b-a)+1},
$$

where $c=c(v)$.

Proof Let $F(v, a, b, d)=\int_{a}^{b} u^{v} e^{-u} d u$. Since $F(v, a, b, d)=d^{-v-1} F(v, a d, b d, 1)$ it is enough to prove the lemma for $d=1$. Assume that $b \geqslant 1$. Then

$$
\begin{aligned}
\int_{a}^{b} u^{v} e^{-u} d u & =e^{-a} \int_{0}^{b-a}(a+u)^{v} e^{-u} d u \\
& \approx e^{-a} \int_{0}^{b-a}\left(a^{v}+u^{v}\right) e^{-u} d u \stackrel{c}{\approx} e^{-a}\left(a^{v}+1\right)((b-a) \wedge 1),
\end{aligned}
$$


which is an equivalent form of Eq. 28 in the case $b \geqslant d=1$. If $b<1$ then

$$
\int_{a}^{b} u^{v} e^{-u} d u \stackrel{c}{\approx} b^{v}(b-a)
$$

which is exactly Eq. 28 in the case $b<d=1$. Note that in all comparisons above the constant $c$ is dependent only on $v$.

Lemma 13 Let $0 \leqslant a \leqslant 1$. Then for every $v>0$ we have

$$
\int_{0}^{a} \frac{e^{-v u} u d u}{\log ^{2} u+1} \approx \frac{1}{(v+1 / a)^{2}\left(\log ^{2}(v+1 / a)+1\right)} .
$$

If additionally av $\leqslant 1$ then

$$
\int_{a}^{1} \frac{e^{-v u} u d u}{1-\log u} \approx \frac{1-a}{(v+1)^{3 / 2}(1+\log (v+1))} .
$$

Proof Let

$$
J(a, v)=\int_{0}^{a} \frac{e^{-v u} u d u}{\log ^{2} u+1} .
$$

First, assume that $a v<2$ then

$$
J(a, v) \approx \int_{0}^{a} \frac{u d u}{\log ^{2} u+1} \leqslant \frac{a^{2}}{2\left(\log ^{2} a+1\right)} .
$$

If additionally $a>1 / 2$ then

$$
J(a, v) \approx 1
$$

If $a \leqslant 1 / 2$ then

$$
J(a, v) \geqslant e^{-2} \int_{a / 2}^{a} \frac{u d u}{\log ^{2} u+1} \geqslant c \frac{a^{2}}{\log ^{2} a+1},
$$

which ends the proof (29) in the case $a v<2$.

We assume now that $a v \geqslant 2$. Observe that for every $q, r \in \mathbb{R}$, from the fact that $\left(q r / \sqrt{r^{2}+1}-\sqrt{r^{2}+1}\right)^{2} \geqslant 0$ we get

$$
(q-r)^{2}+1 \geqslant \frac{q^{2}}{r^{2}+1} .
$$

This implies that

$$
\frac{1}{(\log v-\log s)^{2}+1} \leqslant \frac{\log ^{2} s+1}{\log ^{2} v}
$$

Consequently, we obtain

$$
J(a, v)=\frac{1}{v^{2}} \int_{0}^{a v} \frac{e^{-s} s d s}{(\log v-\log s)^{2}+1} \leqslant \frac{1}{v^{2} \log ^{2} v} \int_{0}^{\infty} e^{-s} s\left(\log ^{2} s+1\right) d s .
$$

On the other hand we get

$$
J(a, v)=\frac{1}{v^{2}} \int_{0}^{a v} \frac{e^{-s} s d s}{(\log v-\log s)^{2}+1} \geqslant \frac{1}{v^{2}\left(\log ^{2} v+1\right)} \int_{1}^{2} e^{-s} s d s,
$$

which ends the proof (Eq. 29). 
Let

$$
\begin{aligned}
J(a, v) & =\int_{a}^{1} \frac{e^{-v u} u^{1 / 2}}{1-\log u} d u \\
& =\frac{1}{v^{3 / 2}} \int_{v a}^{v} \frac{e^{-s} s^{1 / 2}}{1-\log s+\log v} d s .
\end{aligned}
$$

If $v<2$ then

$$
J(a, v) \approx \int_{a}^{1} \frac{u^{1 / 2}}{1-\log u} d u \approx 1-a,
$$

which is Eq. 30 in this case.

Next, we assume that $2 \leqslant v \leqslant 1 / a$. Using the fact that for every $r>q>0$ we have $r-q+1 \geqslant r /(q+1)$ we get

$$
\begin{aligned}
\int_{v a}^{v} \frac{e^{-s} s^{1 / 2}}{1-\log s+\log v} d s & \leqslant\left(\int_{0}^{1}+\int_{1}^{v}\right) \frac{e^{-s} s^{1 / 2}}{1-\log s+\log v} d s \\
& \leqslant \frac{c}{\log v} \int_{0}^{1} e^{-s} s^{1 / 2} d s+\frac{1}{\log v} \int_{1}^{v} e^{-s} s^{1 / 2}(1+\log s) d s \leqslant \frac{c_{2}}{\log v} .
\end{aligned}
$$

Moreover, for $2<v<1 / a$ we get

$$
\int_{v a}^{v} \frac{e^{-s} s^{1 / 2}}{1-\log s+\log v} d s \geqslant \int_{1}^{2} \frac{e^{-s} s^{1 / 2}}{1-\log s+\log v} d s \geqslant \frac{c_{1}}{\log v} .
$$

We have just proved that

$$
J(a, v) \approx \frac{1}{v^{3 / 2} \log v}, 2<v<1 / a .
$$

Combining Eqs. 31 and 32 we complete the proof of Eq. 30.

A.2 Estimates of $w_{1, \lambda}$ and $w_{2, \lambda}$

Lemma 14 There exist constants $c=c(\mu)>0$ and $\theta_{\mu}>0$ such that

$$
\left|w_{1, \lambda}(v)\right| \leqslant c x^{\mu-3 / 2} e^{-v \theta_{\mu}}, \quad v>0 .
$$

Proof Recall that the set of zeros of the function $K_{\mu}(z)$ is denoted by $Z=$ $\left\{z_{1}, \ldots, z_{k_{\mu}}\right\}$. For every $z_{i} \in Z$ we have $\Re z_{i}<0$ and the set $Z$ is finite. Consequently, there exists constant $c_{1}>0$ such that for every $i=1, \ldots, k_{\mu}$ we have

$$
\left|\frac{K_{\mu}\left(x z_{i}\right)}{K_{\mu-1}\left(z_{i}\right)}\right|=\left|\frac{K_{\mu}\left(x z_{i}\right)-K_{\mu}\left(z_{i}\right)}{K_{\mu-1}\left(z_{i}\right)}\right| \leqslant c_{1}(x-1) .
$$

Moreover, using Eq. 6, the constant $c_{1}$ can be chosen to ensure that for every $x \geqslant 2$ we have

$$
\left|\frac{e^{\lambda z_{i}} K_{\mu}\left(x z_{i}\right)}{K_{\mu-1}\left(z_{i}\right)}\right| \leqslant c_{1} \frac{1}{\sqrt{x}} .
$$


Hence there are $c_{2}>0$ and $\theta_{\mu}=-\max _{i}\left\{\Re z_{i}\right\}>0$ such that

$$
\left|\frac{z_{i} e^{\lambda z_{i}} K_{\mu}\left(x z_{i}\right)}{K_{\mu-1}\left(z_{i}\right)} e^{z_{i} v}\right| \leqslant c_{2} \frac{\lambda}{x^{3 / 2}} e^{-\theta_{\mu} v}
$$

and it gives

$$
\left|w_{1, \lambda}(v)\right| \leqslant c_{3} x^{\mu-3 / 2} e^{-\theta_{\mu} v}
$$

Let us define for $u>0$ and $x>1$ :

$$
S_{\mu}(x, u)=I_{\mu}(x u) K_{\mu}(u)-I_{\mu}(u) K_{\mu}(x u) .
$$

The function $S_{\mu}$ appears in the formula for $w_{2, \lambda}$ and consequently, the uniform estimates of the function $S_{\mu}$, which are given in the next Lemma, are crucial to get the estimates of the function $w_{2, \lambda}$ given in Lemmas 16 and 17 for $\mu>0$ and $\mu=0$, respectively.

Lemma 15 For $\mu \geqslant 0$ we have

$$
\frac{\lambda}{x} \frac{K_{\mu}(x u)}{K_{\mu}(u)} \leqslant S_{\mu}(x, u) \leqslant \lambda \frac{K_{\mu}(u)}{K_{\mu}(x u)} .
$$

There are constants $c_{1}$ and $c_{2}$ such that for $\mu \geqslant 0,1<x<2$ and $u>0$ we thus obtain

$$
c_{1} \lambda e^{-\lambda u} \leqslant S_{\mu}(x, u) \leqslant c_{2} \lambda e^{\lambda u} .
$$

There is a constant $c_{1}$ such that for $\mu>0, x>2$ and $u>0$ we have

$$
c_{1} I_{\mu}(x u) K_{\mu}(u) \leqslant S_{\mu}(x, u) \leqslant I_{\mu}(x u) K_{\mu}(u) .
$$

There is a constant $c_{1}$ such that for $\mu=0, x>2$ and $x u>1$ we have

$$
c_{1} I_{0}(x u) K_{0}(u) \leqslant S_{0}(x, u) \leqslant I_{0}(x u) K_{0}(u) .
$$

For $\mu=0, x>2$ and $x u<1$ we have

$$
S_{0}(x, u) \approx \log x .
$$

Proof Write for $u>0$

$$
\psi(u)=\frac{I_{\mu}(u)}{K_{\mu}(u)} .
$$

Then by Eq. 1 we have

$$
\psi^{\prime}(u)=\frac{1}{u} \frac{1}{K_{\mu}^{2}(u)} .
$$

Writing

$$
S_{\mu}(x, u)=[\psi(x u)-\psi(u)] K_{\mu}(x u) K_{\mu}(u) .
$$


we obtain from the Lagrange theorem

$$
S_{\mu}(x, u)=\frac{x u-u}{\theta x u} \frac{K_{\mu}(x u) K_{\mu}(u)}{K_{\mu}(\theta x u) K_{\mu}(\theta x u)} .
$$

The quantity $\theta$ here has the property $1 \leqslant \theta x \leqslant x$. This and the monotonicity of the function $K_{\mu}$, give the estimate (34). The estimate (35) is a direct consequence of the limiting behaviour of the function $K_{\mu}$.

To prove Eqs. 36 and 37 note that the function $g(x, u)=\frac{\psi(u)}{\psi(x u)}=\frac{I_{\mu}(u) K_{\mu}(x u)}{K_{\mu}(u) I_{\mu}(x u)}$ as a function of $x$ is decreasing. Hence for $x>2$,

$$
g(x, u) \leqslant g(2, u)<1, u>0 .
$$

If $\mu>0$, then the limits at 0 and $\infty$ of $g(2, u)$ are strictly less then 1 . By continuity

$$
\sup _{u>0} g(2, u)=a<1 .
$$

If $\mu=0$ by the same argument for $x>2$,

$$
g(x, u) \leqslant \sup _{v \geqslant 1 / 2} g(2, v)<1, u \geqslant 1 / 2 .
$$

If $x u>1, u<1 / 2$ and $x>2$ then

$$
g(x, u)=\frac{I_{0}(u) K_{0}(x u)}{K_{0}(u) I_{0}(x u)} \leqslant \frac{I_{0}(1 / 2) K_{0}(1)}{K_{0}(1 / 2) I_{0}(1)}=g(2,1 / 2)<1 .
$$

These estimates imply that

$$
g(x, u) \leqslant \sup _{v \geqslant 1 / 2} g(2, v)=a<1, u>1 / x .
$$

Hence, in both cases $(\mu=0$ or $\mu>0)$ we have for $x>2$ and $x u>1$

$$
S_{\mu}(x, u)=\left[1-\frac{\psi(u)}{\psi(x u)}\right] \psi(x u) K_{\mu}(x u) K_{\mu}(u) \geqslant(1-a) I_{\mu}(x u) K_{\mu}(u),
$$

which ends the proof of Eqs. 36 and 37.

It remains to consider $\mu=0, x>2$ and $x u<1$. We apply the asymptotics of $K_{0}$ at 0. Namely, by Eq. 4 we can write

$$
K_{0}(z)=-\log \frac{z}{2} I_{0}(z)+A(z),
$$

where $A(z) \rightarrow c>0, z \rightarrow 0$. This yields

$$
S_{0}(x, u)=I_{0}(u x) I_{0}(u) \log x+I_{0}(u x) A(u)-I_{0}(u) A(u x) \approx \log x .
$$

Lemma 16 For $\mu>0$ and $x>1$ we have

$$
w_{2, \lambda}(v) \approx(-\cos (\pi \mu)) \frac{x^{2 \mu-1}}{(v+1)^{\mu+3 / 2}(v+x)^{\mu+1 / 2}}, v>0 .
$$


Proof Let us denote

$$
\begin{aligned}
h(x, u, v) & =\frac{S_{\mu}(x, u)}{\cos ^{2}(\pi \mu) K_{\mu}^{2}(u)+\left(\pi I_{\mu}(u)+\sin (\pi \mu) K_{\mu}(u)\right)^{2}} e^{-\lambda u} e^{-v u} u \\
& \approx \frac{S_{\mu}(x, u)}{K_{\mu}^{2}(u)+I_{\mu}^{2}(u)} e^{-\lambda u} e^{-v u} u .
\end{aligned}
$$

Since $w_{2, \lambda}(v)=-\cos (\pi \mu) \frac{x^{\mu}}{\lambda} \int_{0}^{\infty} h(x, u, v) d u$, it is enough to estimate $\int_{0}^{\infty} h(x, u, v) d u$. which is done below for two cases.

A) Case $1<x<2$.

Suppose that $0<u<1$. By Lemma $15, S_{\mu}(x, u) \approx \lambda$, thus

$$
\begin{aligned}
h(x, u, v) & \approx \frac{\lambda}{K_{\mu}^{2}(u)+I_{\mu}^{2}(u)} e^{-\lambda u} e^{-v u} u \\
& \approx \lambda u^{2 \mu+1} e^{-(v+1) u}, 0<u<1 .
\end{aligned}
$$

For $u>1$ we have, by Lemma $15, S_{\mu}(x, u) \leqslant c \lambda e^{u}$, which yields the following upper bound.

$$
\begin{aligned}
h(x, u, v) & \leqslant c \frac{\lambda e^{u}}{K_{\mu}^{2}(u)+I_{\mu}^{2}(u)} e^{-\lambda u} e^{-v u} u \\
& \leqslant c \lambda u e^{-(v+1) u} .
\end{aligned}
$$

Applying Lemma 12 to the above estimates we arrive at

$$
\int_{0}^{1} h(x, u, v) d u \approx \frac{\lambda}{(v+1)^{2 \mu+2}}
$$

and

$$
\int_{1}^{\infty} h(x, u, v) d u \leqslant c \frac{\lambda}{(v+1)^{2}} e^{-(v+1)} .
$$

Combining both integrals we obtain

$$
\int_{0}^{\infty} h(x, u, v) d u \approx \frac{\lambda}{(v+1)^{2 \mu+2}},
$$

which proves the lemma in the case $1<x<2$.

B) Case $x>2$.

By Lemma 15, $S_{\mu}(x, u) \approx I_{\mu}(x u) K_{\mu}(u)$, which implies

$$
h(x, u, v) \approx \frac{I_{\mu}(x u) K_{\mu}(u)}{K_{\mu}^{2}(u)+I_{\mu}^{2}(u)} e^{-\lambda u} e^{-v u} u .
$$

Next, using the asymptotics of the Bessel functions, we arrive at

$$
h(x, u, v) \approx\left\{\begin{array}{l}
x^{\mu} u^{2 \mu+1} e^{-(v+\lambda) u}, x u<1, \\
x^{-1 / 2} u^{\mu+1 / 2} e^{-v u}, \quad 1 / x<u<1, \\
x^{-1 / 2} u e^{-(v+2) u}, \quad u>1 .
\end{array}\right.
$$


To estimate $H(x, v)=\int_{0}^{\infty} h(x, u, v) d u$ we split the integral into three parts:

$$
\begin{aligned}
H(x, v) & =\int_{0}^{1 / x} h(x, u, v) d u+\int_{1 / x}^{1} h(x, u, v) d u+\int_{1}^{\infty} h(x, u, v) \\
& =J_{1}(x, v)+J_{2}(x, v)+J_{3}(x, v) .
\end{aligned}
$$

Applying Lemma 12 with $a=0, b=1 / x$ and $d=v+\lambda$, the first integral can be estimated in the following way:

$$
\begin{aligned}
J_{1}(x, v) & \approx x^{\mu} \int_{0}^{1 / x} u^{2 \mu+1} e^{-(v+\lambda) u} d u \\
& \approx \frac{x^{\mu}}{(v+\lambda)^{2 \mu+1}} \frac{1 / x}{1+(v+\lambda) / x} \\
& \approx \frac{x^{\mu}}{(v+x)^{2 \mu+2}} .
\end{aligned}
$$

Next, we deal with the second integral. Again, by Lemma 12 with $a=1 / x$, $b=1$ and $d=v$, we obtain

$$
\begin{aligned}
J_{2}(x, v) & \approx x^{-1 / 2} \int_{1 / x}^{1} u^{\mu+1 / 2} e^{-v u} d u \\
& \approx x^{-1 / 2}\left(\frac{\frac{1}{x}+\frac{1}{v}}{1+\frac{1}{v}}\right)^{\mu+1 / 2} e^{-v / x} \frac{1-1 / x}{1+v-v / x} \\
& \approx x^{-1 / 2}\left(\frac{\frac{1}{x}+\frac{1}{v}}{1+\frac{1}{v}}\right)^{\mu+1 / 2} e^{-v / x} \frac{\lambda}{x+\lambda v} \\
& \approx x^{-1 / 2}\left(\frac{1+\frac{v}{x}}{1+v}\right)^{\mu+1 / 2} e^{-v / x} \frac{1}{v+1} .
\end{aligned}
$$

The third integral can be estimated for $v>0$ as follows.

$$
J_{3}(x, v) \approx x^{-1 / 2} \int_{1}^{\infty} u e^{-(v+2) u} d u \approx x^{-1 / 2} \frac{1}{(v+2)} e^{-(v+2)}
$$

It is clear that

$$
J_{3}(x, v) \leqslant c \frac{x^{\mu}}{(v+1)^{\mu+3 / 2}(v+x)^{\mu+1 / 2}} .
$$

Next,

$$
\begin{aligned}
\frac{(v+1)^{\mu+3 / 2}(v+x)^{\mu+1 / 2}}{x^{\mu}} I_{1} & \approx \frac{(v+1)^{\mu+3 / 2}(v+x)^{\mu+1 / 2}}{x^{\mu}} \frac{x^{\mu}}{(v+x)^{2 \mu+2}} \\
& =\frac{(v+1)^{\mu+3 / 2}}{(v+x)^{\mu+3 / 2}}
\end{aligned}
$$


and

$$
\begin{aligned}
& \frac{(v+1)^{\mu+3 / 2}(v+x)^{\mu+1 / 2}}{x^{\mu}} I_{2} \\
& \approx \frac{(v+1)^{\mu+3 / 2}(v+x)^{\mu+1 / 2}}{x^{\mu}} x^{-1 / 2}\left(\frac{1+\frac{v}{x}}{1+v}\right)^{\mu+1 / 2} e^{-v / x} \frac{1}{v+1} \\
& =(1+v / x)^{2 \mu+1} e^{-v / x} .
\end{aligned}
$$

The observation $\frac{(v+1)^{\mu+3 / 2}}{(v+x)^{\mu+3 / 2}}+(1+v / x)^{2 \mu+1} e^{-v / x} \approx 1$ completes the proof.

Lemma 17 For $\mu=0$ and $x>1$ we have

$$
-w_{\lambda}(v) \approx \frac{1}{x(v+1)^{3 / 2}(v+x)^{1 / 2}} \frac{\log (x+1)}{\log (v+2)(\log (x+1)+\log (v+2))}, v>0
$$

Proof Let us denote

$$
f(x, v)=\frac{1}{x(v+1)^{3 / 2}(v+x)^{1 / 2}} \frac{\log (x+1)}{\log (v+2)(\log (x+1)+\log (v+2))} .
$$

We write

$$
\begin{aligned}
\int_{0}^{\infty} \frac{S_{0}(x, u) e^{-\lambda u}}{K_{0}^{2}(u)+\pi^{2} I_{0}^{2}(u)} e^{-v u} u d u & =\left(\int_{0}^{1 / x}+\int_{1 / x}^{1}+\int_{1}^{\infty}\right) \frac{S_{0}(x, u) e^{-\lambda u}}{K_{0}^{2}(u)+\pi^{2} I_{0}^{2}(u)} e^{-v u} u d u \\
& =J_{1}(x, v)+J_{2}(x, v)+J_{3}(x, v) .
\end{aligned}
$$

The estimates of $J_{3}(x, v)$ are exactly the same as the corresponding estimates proved in Lemma 16. Hence, for $x \geqslant 2$,

$$
J_{3}(x, v) \approx \frac{1}{\sqrt{x}} \frac{e^{-(v+2)}}{(v+2)^{2}}, \quad v>0,
$$

and for $1<x<2$,

$$
J_{3}(x, v) \leqslant c \frac{\lambda}{(v+1)^{2}} e^{-(v+1)} .
$$

To estimate $J_{2}(x, v)$ for $x>2$ observe that for $x u \geqslant 1$ and $u<1, S_{0}(x, u) \approx$ $\frac{e^{x u}}{\sqrt{x u}} K_{0}(u) \approx \frac{e^{x u}}{\sqrt{x u}}(1-\log u)$. This follows from the asymptotic expansions for $I_{0}$ and $K_{0}$ (see Eqs. 2 and 3), and Lemma 15. Thus

$$
\begin{aligned}
J_{2}(x, v) & \approx \frac{1}{x^{1 / 2}} \int_{1 / x}^{1} \frac{K_{0}(u) e^{u} e^{-v u} u^{1 / 2}}{K_{0}^{2}(u)+\pi^{2} I_{0}^{2}(u)} d u \approx \frac{1}{x^{1 / 2}} \int_{1 / x}^{1} \frac{e^{-v u} u^{1 / 2}}{1-\log u} d u \\
& \approx \frac{1}{x^{1 / 2}} \frac{1-1 / x}{(v+1)^{3 / 2}(1+\log (v+1))}, v \leqslant x
\end{aligned}
$$


where the last step is a consequence of Eq. 30 with $a=1 / x$. Next,

$$
\begin{aligned}
J_{2}(x, v) & \approx \frac{1}{x^{1 / 2}} \int_{1 / x}^{1} \frac{K_{0}(u) e^{u} e^{-v u} u^{1 / 2}}{K_{0}^{2}(u)+\pi^{2} I_{0}^{2}(u)} d u \leqslant c \frac{1}{x^{1 / 2}} \int_{0}^{1} \frac{e^{-v u} u^{1 / 2}}{1-\log u} d u \\
& \leqslant c \frac{1}{x^{1 / 2}} \frac{1}{(v+1)^{3 / 2}(1+\log (v+1))}, v>0,
\end{aligned}
$$

where, again, the last step is a consequence of Eq. 30 with $a=0$.

For $x<2$ and $1 / x \leqslant u<1$, by Lemma 15 , we have $S_{0}(x, u) \approx \lambda$ hence

$$
J_{2}(x, v) \approx \lambda \int_{1 / x}^{1} \frac{e^{-v u} u}{K_{0}^{2}(u)+\pi^{2} I_{0}^{2}(u)} d u \approx \lambda \int_{1 / x}^{1} \frac{e^{-v u} u}{1+\log ^{2} u} d u
$$

Finally, for $0<u<1 / x$ we have, by Lemma $15, S_{0}(x, u) \approx \log x, x>0$ and consequently using Eq. 29, with $a=1 / x$, we obtain

$$
\begin{aligned}
J_{1}(x, v) & \approx \log x \int_{0}^{1 / x} \frac{e^{-\lambda u} e^{-v u} u}{K_{0}^{2}(u)+\pi^{2} I_{0}^{2}(u)} d u \approx \log x \int_{0}^{1 / x} \frac{e^{-v u} u}{1+\log ^{2} u} d u . \\
& \approx \frac{\log x}{(v+x)^{2}\left(\log ^{2}(v+x)+1\right)} .
\end{aligned}
$$

We are now ready to estimate the function $-w_{\lambda}(v)$. At first we consider $1<x<2$. Taking into account that $\log x \approx \lambda$, using Eqs. 44 and $45 \mathrm{w}$ arrive at

$$
\begin{aligned}
J_{1}(x, v)+J_{2}(x, v) & \approx \lambda \int_{0}^{1} \frac{e^{-v u} u}{1+\log ^{2} u} d u \\
& \approx \frac{\lambda}{(v+1)^{2}\left(\log ^{2}(v+1)+1\right)} .
\end{aligned}
$$

Combining this with Eq. 41 we have for $1<x<2$,

$$
\begin{aligned}
-w_{\lambda}(v) & =\frac{1}{\lambda}\left(J_{1}(x, v)+J_{2}(x, v)+J_{3}(x, v)\right) \approx \frac{1}{\lambda}\left(J_{1}(x, v)+J_{2}(x, v)\right) \\
& \approx \frac{1}{(v+1)^{2}\left(\log ^{2}(v+1)+1\right)} \approx f(x, v) .
\end{aligned}
$$

Taking into account Eqs. 40, 42 and 46, we infer that for $0<v<2$ and $x>2$, $J_{1}(x, v)+J_{2}(x, v) \leqslant c J_{3}(x, v)$, which yields

$$
-w_{\lambda}(v)=\frac{1}{\lambda}\left(J_{1}(x, v)+J_{2}(x, v)+J_{3}(x, v)\right) \approx \frac{1}{\lambda} J_{3}(x, v) \approx \frac{1}{x^{3 / 2}} \approx f(x, v) .
$$

For $2 \leqslant v<x$, by Eqs. 46, 40 and $42, J_{1}(x, v)+J_{3}(x, v) \leqslant c J_{2}(x, v)$. Hence,

$$
-w_{\lambda}(v)=\frac{1}{\lambda}\left(J_{1}(x, v)+J_{2}(x, v)+J_{3}(x, v)\right) \approx \frac{1}{\lambda} J_{2}(x, v) \approx \frac{1}{x^{3 / 2} v^{3 / 2} \log v} \approx f(x, v) .
$$


Finally for $v \geqslant x>2$, by Eqs. 43, 46 and 40 we have $J_{2}(x, v)+J_{3}(x, v) \leqslant c J_{1}(x, v)$ for some constant $c>1$ and consequently

$$
-w_{\lambda}(v)=\frac{1}{\lambda}\left(J_{1}(x, v)+J_{2}(x, v)+J_{3}(x, v)\right) \approx \frac{1}{\lambda} J_{1}(x, v) \approx \frac{\log x}{x v^{2} \log ^{2} v} \approx f(x, v) .
$$

The proof is completed.

\section{References}

1. Abramowitz, M., Stegun, I.A.: Handbook of Mathematical Functions with Formulas, Graphs, and Mathematical Tables, 9th edn. Dover, New York (1972)

2. Borodin, A.N., Salminen, P.: Handbook of Brownian Motion-Facts and Formulae, 2nd edn. Birkhauser, Basel (2002)

3. Byczkowski, T., Graczyk, P., Stos, A.: Poisson kernels of half-spaces in real hyperbolic spaces. Rev. Mat. Iberoam. 23(1), 85-126 (2007)

4. Byczkowski, T., Małecki, J., Ryznar, M.: Hitting Half-spaces by Bessel-Brownian diffusions. Potential Anal. 33, 47-83 (2010). arXiv:math.PR/0612176

5. Byczkowski, T., Ryznar, M.: Hitting distibution of geometric Brownian motion. Stud. Math. 173(1), 19-38 (2006)

6. Collete, P., Martinez, S., San Martin, J.: Asymptotic behaviour of a Brownian motion on exterior domains. Probab. Theory Relat. Fields 116, 303-316 (2000)

7. Davies, E.B.: Heat kernels and spectral theory. In: Cambridge Tracts in Mathematics, vol. 92. Cambridge University Press, Cambridge (1990)

8. Erdelyi, A., et al.: Tables of Integral Transforms, vols. I and II. McGraw-Hill, New York (1954)

9. Getoor, R.K., Sharpe, M.J.: Excursions of Brownian motion and Bessel processes. Z. Wahrscheinlichkeitstheor. Verw. Geb. 47, 83-106 (1979)

10. Grigor'yan, A., Saloff-Coste, L.: Hitting probabilities for Brownian motion on Riemannian manifolds. J. Math. Pure Appl. 81, 115-142 (2002)

11. Gruet, J.-C.: Semi-groupe du mouvement Brownien hyperbolique. Stoch. Stoch. Rep. 56, 53-61 (1996)

12. Hunt, G.A.: Some theorems concerning brownian motion. Trans. Am. Math. Soc. 81, 294-319 (1956)

13. Itô, K., McKean Jr., H.P.: Diffusion Processes and Their Sample Paths. Springer, New York (1974)

14. Kent, J.T.: Some probabilistic properties of Bessel functions. Ann. Probab. 6, 760-770 (1978)

15. Lamperti, J.: Semi-stable Markov processes. Z. Wahrscheinlichkeitstheor. Verw. Geb. 22, 205-225 (1972)

16. Matsumoto, H., Yor, M.: Exponential functionals of Brownian motion, I: probability laws at fixed time. Probab. Surv. 2, 312-347 (2005)

17. Matsumoto, H., Yor, M.: Exponential functionals of Brownian motion, II: some related diffusion processes. Probab. Surv. 2, 348-384 (2005)

18. McKean, H.P.: The Bessel motion and a singular integral equation. Mem. Sci. Univ. Kyoto, Ser A, Math. 33, 317-322 (1960)

19. Molchanov, S.A., Ostrowski, S.A.: Symmetric stable processes as traces of degenerate diffusion processes. Theory Probab. Appl. 12, 128-131 (1969)

20. Port, S.C.: Hitting times for transient stable process. Pac. J. Math. 21, 161-165 (1967)

21. Revuz, D., Yor, M.: Continuous Martingales and Brownian Motion. Springer, New York (1999)

22. Uchiyama, K.: Asymptotic estimates of the distribution of brownian hitting time of a disc. J. Theor. Probab. 25(2), 450-463 (2012)

23. Yor, M.: Some Aspects of Brownian Motion, Part I: Some Special Functional. Birkhaäuser, Basel (1992)

24. Yor, M.: Exponential Functionals of Brownian Motion and Related Processes. Springer Finance, Berlin (2001) 